\title{
A validation and code-to-code verification of FAST for a megawatt-scale wind turbine with aeroelastically tailored blades
}

\author{
Srinivas Guntur ${ }^{1, a}$, Jason Jonkman ${ }^{1}$, Ryan Sievers ${ }^{2}$, Michael A. Sprague ${ }^{1}$, Scott $_{\text {Schreck }}{ }^{1}$, and \\ Qi Wang ${ }^{1, a}$ \\ ${ }^{1}$ National Renewable Energy Laboratory, Golden, CO, USA \\ ${ }^{2}$ Siemens Wind Power, Inc., Boulder, CO, USA \\ ${ }^{a}$ currently at: Siemens Wind Power, Inc., Boulder, CO, USA
}

Correspondence to: Srinivas Guntur (srinivaskguntur@gmail.com)

Received: 1 November 2016 - Discussion started: 6 March 2017

Revised: 17 July 2017 - Accepted: 27 July 2017 - Published: 29 August 2017

\begin{abstract}
This paper presents validation and code-to-code verification of the latest version of the U.S. Department of Energy, National Renewable Energy Laboratory wind turbine aeroelastic engineering simulation tool, FAST v8. A set of 1141 test cases, for which experimental data from a Siemens $2.3 \mathrm{MW}$ machine have been made available and were in accordance with the International Electrotechnical Commission 61400-13 guidelines, were identified. These conditions were simulated using FAST as well as the Siemens in-house aeroelastic code, BHawC. This paper presents a detailed analysis comparing results from FAST with those from BHawC as well as experimental measurements, using statistics including the means and the standard deviations along with the power spectral densities of select turbine parameters and loads. Results indicate good agreement among the predictions using FAST, BHawC, and experimental measurements. These agreements are discussed in detail in this paper, along with some comments regarding the differences seen in these comparisons relative to the inherent uncertainties in such a model-based analysis.
\end{abstract}

\section{Introduction}

It is well known that the wind energy industry is growing rapidly worldwide, and increasingly larger blades are being employed by state-of-the-art wind turbine technology. With the advent of large, flexible blades, the tools required to design and analyze these turbines also need to be updated according to the current and future state of affairs. A wind turbine aeroelastic simulation code consists of several modules that model different aspects of wind turbine physics (aerodynamics, structures, pitch/torque control modules) (Hansen, 2000; Zhang and Huang, 2011). Each of these modules is based on a set of assumptions that may or may not be valid as the size and flexibility of the turbine increases. For example, torsional degree of freedom (DOF) becomes increasingly important for larger, more flexible blades, especially those that use sweep-twist or bend-twist coupling for passive load al- leviation (Rasmussen et al., 2003; Zhang and Huang, 2011). The next generation of aeroelastic codes should be capable of modeling such structures. The latest version of FAST incorporates some of these advancements, as explained later in the paper.

Although the availability of models that can capture physics relevant to state-of-the-art wind turbines is one challenge, the other equally important challenge is validation of such codes. Historically, there have been several international industrial as well as academic efforts to carry out codeto-code verification and validation of wind turbine models against experiments; most notable, among others, are the MEXICO (Schepers et al., 2012, 2014) and the National Renewable Energy Laboratory (NREL) UAE Phase VI (Simms et al., 2001) blind test comparisons. These experiments offered the response of scaled, experimental wind turbines in controlled conditions in wind tunnels. Although these test 
Table 1. International Electrotechnical Commission (IEC) matrix populated with data bins defined by mean wind speed and turbulence intensity. The numbers in the table represent the number of 10 min data sets within each bin, for a total of 1141 cases.

\begin{tabular}{|c|c|c|c|c|c|c|c|c|c|c|c|c|c|c|c|}
\hline \multirow{2}{*}{$\begin{array}{l}\text { Turbulence } \\
\text { Intensity (\%) }\end{array}$} & \multicolumn{15}{|c|}{ Mean wind speed $\left(\mathrm{ms}^{-1}\right)$} \\
\hline & $3.5-4.5$ & $4.5-5.5$ & $5.5-6.5$ & $6.5-7.5$ & $7.5-8.5$ & $8.5-9.5$ & $9.5-10.5$ & $10.5-11.5$ & $11.5-12.5$ & $12.5-14$ & $14-16$ & $16-18$ & $18-20$ & $20-22$ & $22-24$ \\
\hline$<3$ & 0 & 4 & 6 & 3 & 0 & 0 & 0 & 0 & 0 & 0 & 0 & 0 & 0 & 0 & 0 \\
\hline $3-5$ & 0 & 20 & 19 & 19 & 9 & 5 & 1 & 1 & 0 & 0 & 0 & 0 & 0 & 0 & 0 \\
\hline $5-7$ & 0 & 32 & 50 & 32 & 14 & 3 & 7 & 3 & 2 & 2 & 0 & 0 & 0 & 0 & 0 \\
\hline $7-9$ & 0 & 43 & 39 & 44 & 17 & 17 & 3 & 7 & 2 & 2 & 2 & 0 & 1 & 0 & 1 \\
\hline $9-11$ & 0 & 32 & 42 & 35 & 29 & 13 & 7 & 11 & 9 & 5 & 9 & 5 & 0 & 0 & 1 \\
\hline $11-13$ & 0 & 22 & 21 & 16 & 16 & 9 & 12 & 15 & 16 & 14 & 15 & 6 & 4 & 1 & 0 \\
\hline $13-15$ & 0 & 2 & 16 & 25 & 10 & 6 & 14 & 15 & 24 & 11 & 15 & 14 & 5 & 1 & 0 \\
\hline $15-17$ & 0 & 1 & 10 & 11 & 6 & 4 & 7 & 12 & 12 & 10 & 18 & 8 & 9 & 2 & 0 \\
\hline $17-19$ & 0 & 2 & 12 & 6 & 0 & 2 & 3 & 12 & 4 & 10 & 8 & 7 & 5 & 0 & 0 \\
\hline $19-21$ & 0 & 2 & 3 & 3 & 2 & 1 & 3 & 1 & 4 & 2 & 3 & 1 & 0 & 0 & 0 \\
\hline $21-23$ & 0 & 0 & 0 & 1 & 0 & 0 & 0 & 0 & 0 & 1 & 0 & 0 & 0 & 0 & 0 \\
\hline Sum & 0 & 160 & 218 & 195 & 103 & 60 & 57 & 77 & 73 & 57 & 70 & 41 & 24 & 4 & 2 \\
\hline
\end{tabular}

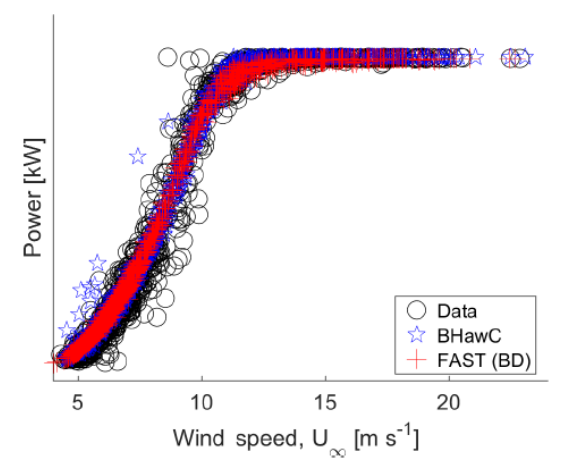

(a)

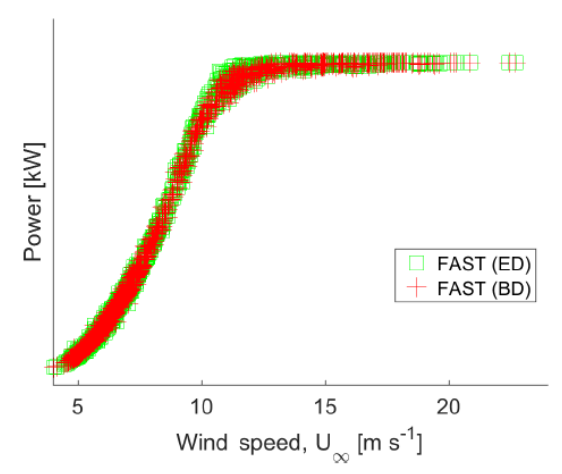

(c)

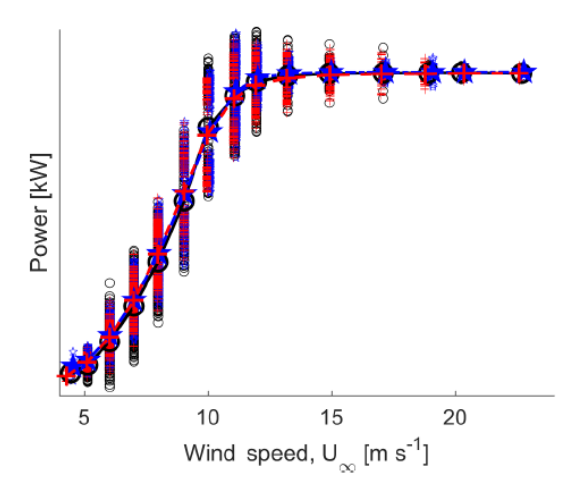

(b)

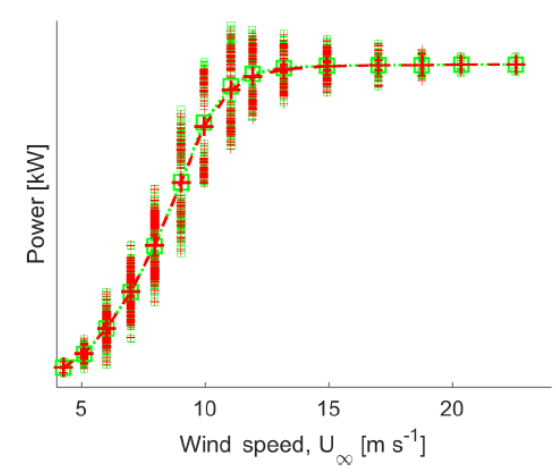

(d)

Figure 1. Electrical power. (a) Individual 10 min averages from experimental data, BHawC, and FAST (BD). (b) Binned averages of data in (a) are denoted by points connected by lines; the scatter above and below these data denotes plus or minus 1 standard deviation, respectively, of the individual $10 \mathrm{~min}$ data sets in that bin. (c) Individual 10 min averages from FAST (BD) and FAST (ED). (d) Binned averages of data in (c) are denoted by the points connected by lines; the scatter above and below these data denotes plus or minus 1 standard deviation, respectively, of the individual $10 \mathrm{~min}$ data sets in that bin.

campaigns have provided high-quality data capturing various aerodynamic effects, structurally these turbines were stiff.

Published works on the validation of high-fidelity aeroelastic codes using larger, flexible turbines, such as (Kallesoe and Kragh, 2016), are rare mainly due to the high costs and physical challenges associated with such an endeavor. To the authors' knowledge, full system validation of wind turbine aeroelastic codes for a wide range of realistic operating conditions, as recommended in (Guzman and Cheng, 2016), for example, is extremely rare to find in published literature despite their relevance to the state of the art in wind turbine research and development. The current work, which has been the result of a research collaboration between NREL and Siemens Energy over a period of 3 years, presents the full 


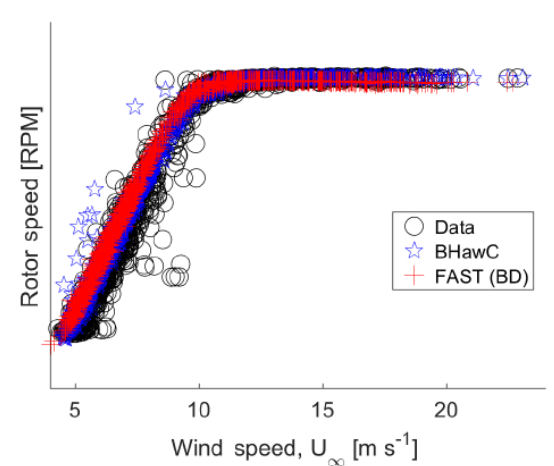

(a)

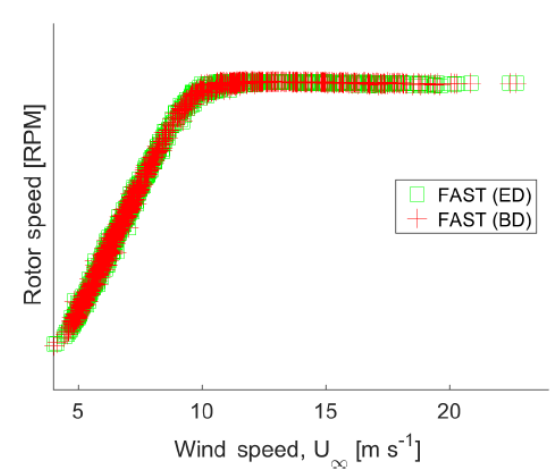

(c)

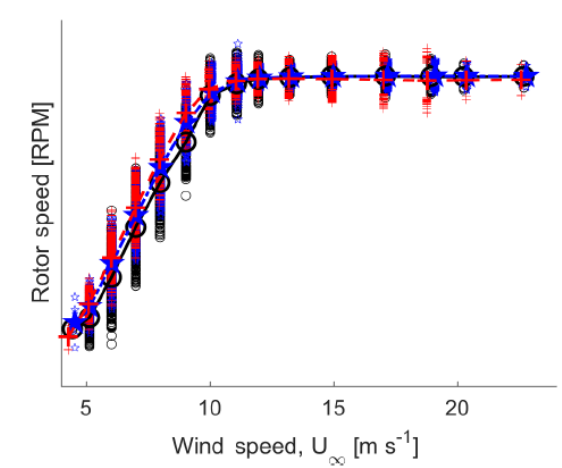

(b)

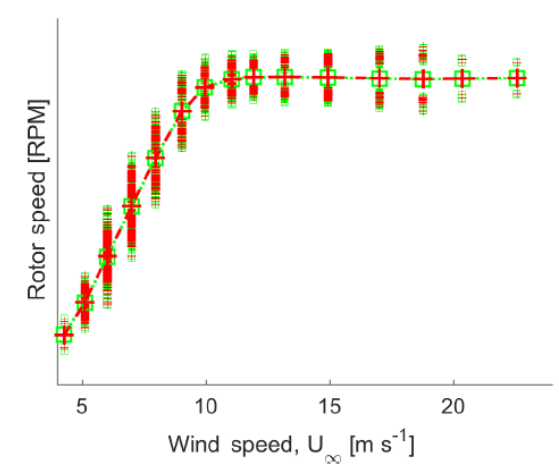

(d)

Figure 2. Rotor speed. (a) Individual 10 min averages from experimental data, BHawC, and FAST (BD). (b) Binned averages of data in (a) are denoted by points connected by lines; the scatter above and below these data denotes plus or minus 1 standard deviation, respectively, of the individual $10 \mathrm{~min}$ data sets in that bin. (c) Individual 10 min averages from FAST (BD) and FAST (ED). (d) Binned averages of data in (c) are denoted by the points connected by lines; the scatter above and below these data denotes plus or minus 1 standard deviation, respectively, of the individual $10 \mathrm{~min}$ data sets in that bin.

system validation of FAST, using detailed and high-quality experimental measurements on a 2.3 MW machine with flexible, aeroelastically tailored blades.

\subsection{FAST v8}

FAST is an open-source multi-physics simulation tool that was created for design and analysis of advanced land-based and offshore wind technology. Underpinning FAST is a modularization framework that enables coupling of various modules, each representing different physics domains of the wind system (Jonkman, 2013; Sprague et al., 2015). FAST version 8 (FAST v8) includes a mesh-mapping utility allowing each module to be independently discretized in space and time and a mathematically rigorous solution procedure supporting loose coupling of modules with implicit-coupling relations. For land-based turbine simulations, FAST has modules for wind inflow (InflowWind); aerodynamics (AeroDyn); control and electrical-drive dynamics (ServoDyn); and blade, drivetrain, nacelle, tower, and platform structural dynamics (ElastoDyn).

In the newest release of FAST, BeamDyn (Wang et al., 2015), a new blade-dynamics module, has been introduced and the AeroDyn aerodynamics module has been overhauled (Ning et al., 2015) to support the analysis of advanced aeroelastically tailored blades. BeamDyn is based on geometrically exact beam theory and is implemented using Legendre spectral finite elements. The geometric nonlinearities, including large displacements and rotations in a three-dimensional space, are captured by BeamDyn without introducing ad hoc assumptions and have initially curved and twisted reference lines. Specifically, BeamDyn supports built-in curve, sweep, and sectional offsets of wind turbine blades. Moreover, along with a cross-sectional analysis tool like VABS (Wang and Yu, 2011) and BECAS (Blasques et al., 2016), BeamDyn is capable of simulating the elastic deformations (extension, shear, bending, and torsion) and the coupling effects between all six DOFs for both isotropic and composite slender structures. When these advanced features are needed, BeamDyn replaces the more simplified blade structural model of ElastoDyn, which is only applicable to straight isotropic blades dominated by bending.

Finally, in the latest FAST v8, AeroDyn has been overhauled to (1) fix underlying problems with the original theoretical treatments, (2) introduce improved skewed-wake and unsteady aerodynamics models, (3) enable the modeling 


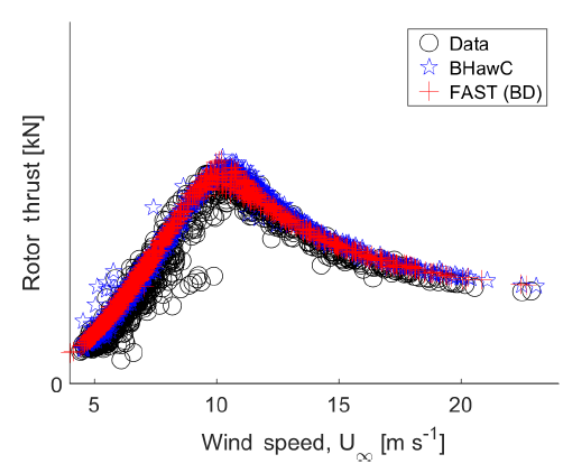

(a)

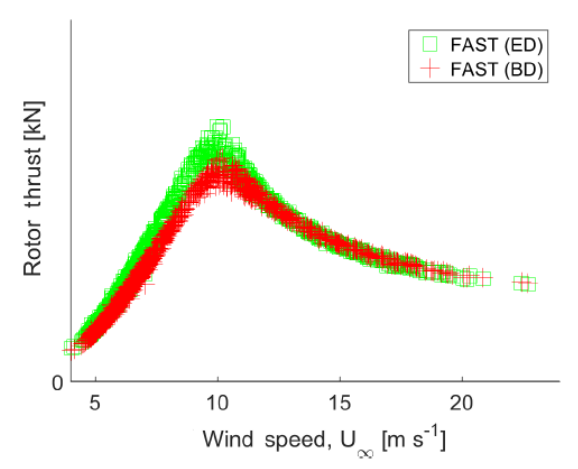

(c)

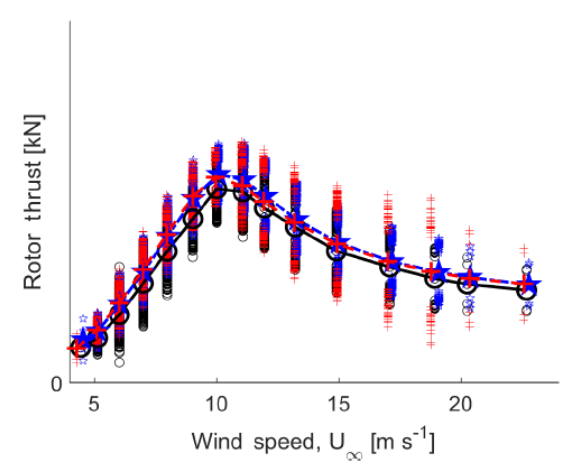

(b)

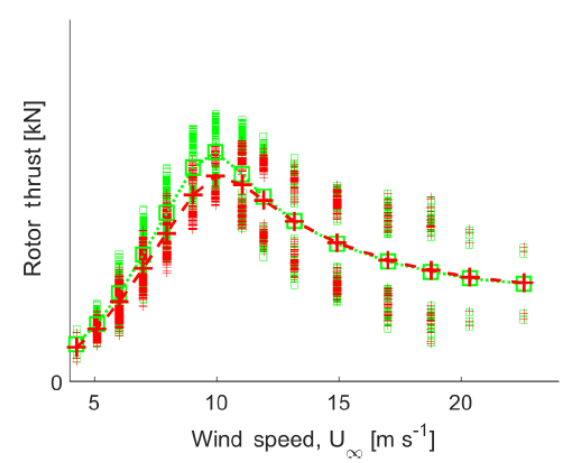

(d)

Figure 3. Rotor thrust force. (a) Individual 10 min averages from experimental data, BHawC, and FAST (BD). (b) Binned averages of data in (a) are denoted by points connected by lines; the scatter above and below these data denotes plus or minus 1 standard deviation, respectively, of the individual $10 \mathrm{~min}$ data sets in that bin. (c) Individual $10 \mathrm{~min}$ averages from FAST (BD) and FAST (ED). (d) Binned averages of data in (c) are denoted by the points connected by lines; the scatter above and below these data denotes plus or minus 1 standard deviation, respectively, of the individual $10 \mathrm{~min}$ data sets in that bin.

of highly flexible and non-straight blades, and (4) support the unique features of the FAST modularization framework (Ning et al., 2015; Damiani et al., 2016).

\subsection{Experimental data}

The National Wind Technology Center (NWTC) at NREL is home to several megawatt-scale test turbines. One of these is a Siemens 2.3 MW machine (SWT-2.3-108) that operates in an upwind configuration and is equipped with a threebladed, $108 \mathrm{~m}$ diameter rotor (Medina et al., 2012). It is a variable-speed turbine operating under a collective pitch control, and the rotor is connected to the generator via a gearbox. The blades used on this machine have a pre-bent geometry (the blade shape under no-load conditions is not straight, but curved). Additionally, these blades are flexible and aeroelastically tailored, incorporating bend-twist coupling. This turbine is instrumented with fiber Bragg strain sensors capable of measuring blade-tip deflections; surface-pressure taps; strain gages at the blade root, tower top, and the tower base; and a data-acquisition system that records turbine operating data such as the rotor speed and electrical power. Wind speed and direction are measured by sensors mounted on an upwind meteorological tower (met tower). Measurements on this heavily instrumented turbine have been collected and shared as part of a cooperative research project between NREL and Siemens Energy. This unique set of measurements is well suited for validation of high-fidelity blade structural modeling tools, like BeamDyn, and facilitates a wide variety of research possibilities. In the current work, the comparisons between FAST and BHawC predictions and the measurements will be discussed for various turbine operating conditions consistent with the recommendations in the International Electrotechnical Commission (IEC) 61400-13 standard (IEC 61400-13, 2001).

\subsection{Current work}

This paper presents a detailed validation and code-to-code verification of the latest version of the U.S. Department of Energy's open-source wind turbine aeroelastic simulation tool FAST v8, which is supported by NREL. A previous paper (Guntur et al., 2016) presented preliminary results from comparisons among predictions from FAST, the Siemens inhouse aeroelastic code BHawC, and the experimental data from the Siemens $2.3 \mathrm{MW}$ wind turbine. Following the pre- 


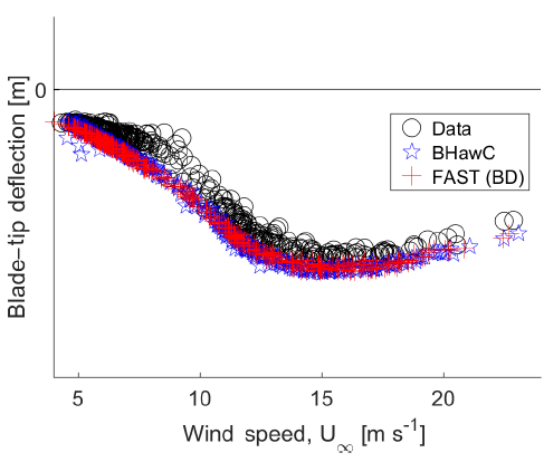

(a)

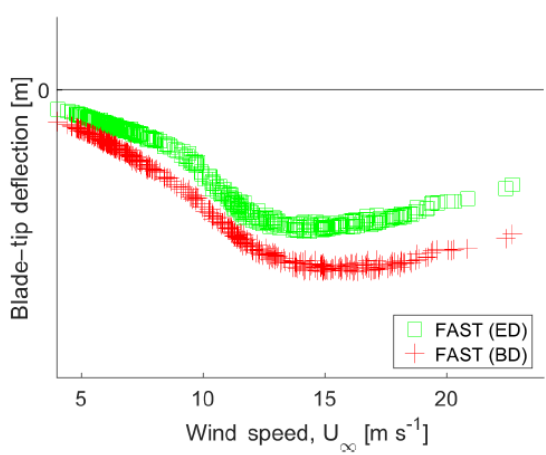

(c)

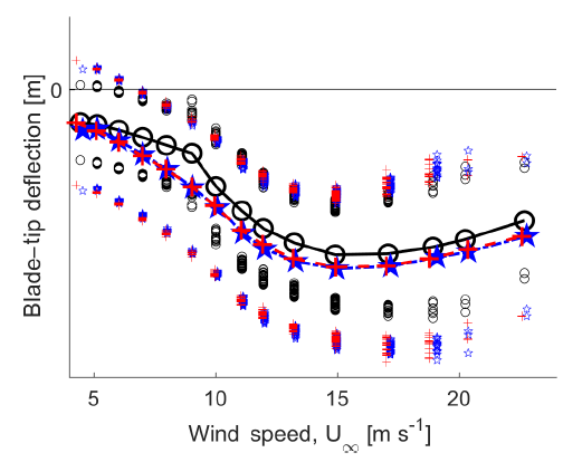

(b)

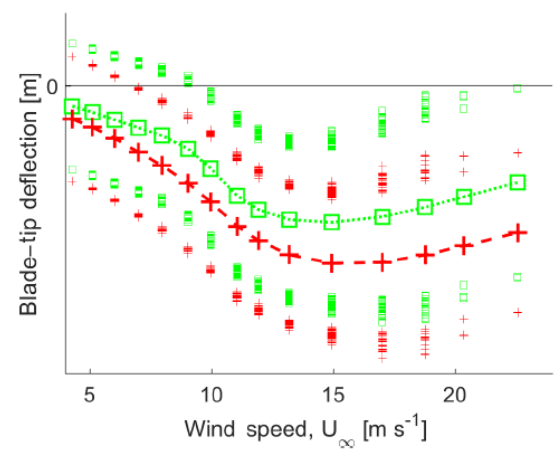

(d)

Figure 4. Blade in-plane tip deflections. (a) Individual 10 min averages from experimental data, BHawC, and FAST (BD). (b) Binned averages of data in (a) are denoted by points connected by lines; the scatter above and below these data denotes plus or minus 1 standard deviation, respectively, of the individual $10 \mathrm{~min}$ data sets in that bin. (c) Individual 10 min averages from FAST (BD) and FAST (ED). (d) Binned averages of data in (c) are denoted by the points connected by lines; the scatter above and below these data denotes plus or minus 1 standard deviation, respectively, of the individual 10 min data sets in that bin.

liminary analysis presented in the previous study, FAST has undergone some improvements, which are highlighted in this paper. This paper presents a validation and code-to-code verification of FAST v8 using results from the improved FAST model analyzed in time as well as frequency domains.

\section{Methods}

The analysis presented in this paper is centered on the Siemens $2.3 \mathrm{MW}$ turbine with a $108 \mathrm{~m}$ rotor that is installed at NREL's NWTC. The turbine is heavily instrumented, and measurement data have been made available through collaborative research between NREL and Siemens Energy. Data were collected over a period of several months under normal operating conditions for a range of wind speeds and turbulence intensities, and these data were used in this validation exercise. Validation was conducted following the guidelines stipulated in the IEC wind turbine load-measurement standard (IEC 61400-13, 2001). Code-to-code verification was accomplished by comparing FAST v8 simulation results with those of the Siemens in-house aeroelastic simulation tool, BHawC.

\subsection{Experimental measurements}

A large amount of test data were recorded on the Siemens 2.3 MW wind turbine and the NWTC $135 \mathrm{~m}$ met tower. The met tower is located approximately 2.5 rotor diameters upstream of the turbine and is instrumented with several sensors along its length that measure the wind speed, wind direction, and atmospheric pressure. The inflow wind speed data used in this analysis were recorded at $80 \mathrm{~m}$, which is close to the turbine hub height. Each 10 min data set contains surface-pressure measurement data, strain-gage data, turbine-operation data, and the inflow data from the met tower. In total, several months of data have been recorded, of which this report presents data from $114110 \mathrm{~min}$ data sets. The data samples were selected based on their mean hub-height wind speed and turbulence intensities in order to populate the recommended test matrix of the IEC 61400-13 (see Table 1). While the 1141 simulations were run according to Table 1, the results plotted in Sect. 3 are binned only by wind speed. Overall, these data represent operating conditions with inflow velocities between the turbine's cut-in and the cut-out wind speeds, at various inflow turbulence intensity levels, up to approximately $23 \%$. 


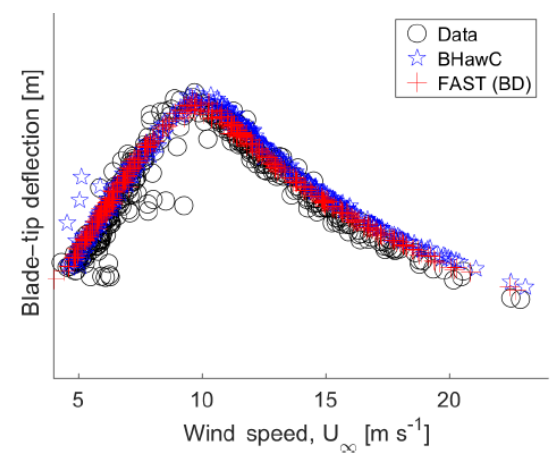

(a)

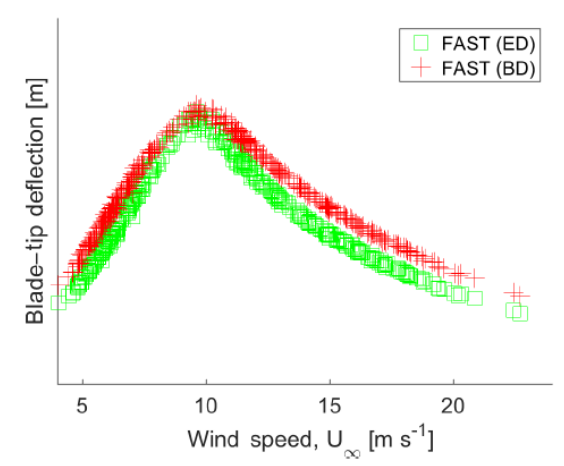

(c)

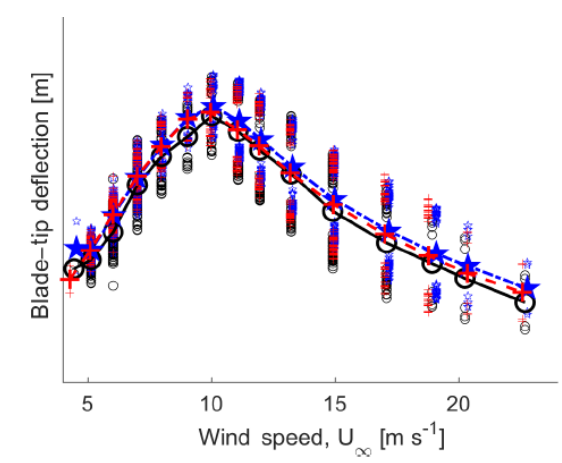

(b)

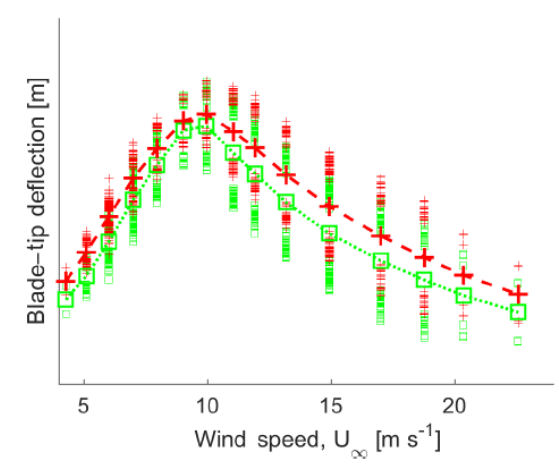

(d)

Figure 5. Blade out-of-plane tip deflections. (a) Individual 10 min averages from experimental data, BHawC, and FAST (BD). (b) Binned averages of data in (a) are denoted by points connected by lines; the scatter above and below these data denotes plus or minus 1 standard deviation, respectively, of the individual $10 \mathrm{~min}$ data sets in that bin. (c) Individual 10 min averages from FAST (BD) and FAST (ED). (d) Binned averages of data in (c) are denoted by the points connected by lines; the scatter above and below these data denotes plus or minus 1 standard deviation, respectively, of the individual $10 \mathrm{~min}$ data sets in that bin.

Each bin in the test matrix (Table 1) contains several 10 min data sets. For each data set, the mean, standard deviation, and the power spectral density (PSD) of the following quantities of interest (QOIs) are determined from both the simulations and the measured data:

- rotor speed

- electrical power

- blade-root bending moments (in and out of the rotor plane)

- main-shaft bending moments (yaw and tilt directions, in a nonrotating coordinate system)

- tower-top torsional moment

- tower-bottom bending moments (parallel and perpendicular to the mean wind direction)

- blade-tip deflections (in and out of the rotor plane).

For brevity, only select PSDs are presented in this paper at three different operating conditions for each QOI: one at below-rated, one at rated, and one at above-rated hub-height inflow wind speed.

\subsection{Siemens aeroelastic simulation tool BHawC}

BHawC is the Siemens in-house aeroelastic simulation tool used to study the dynamic response of wind turbines. The model consists of substructures for foundation, tower, nacelle, drivetrain, gearbox, hub, and blades. The structure is modeled primarily with finite beam elements, and the aerodynamics is modeled using blade element momentum theory. The code is coupled to a controller identical to that on the real turbine.

The structural model of BHawC employs a co-rotational beam formulation, which is a combined multi-body and linear finite-element representation allowing for geometric nonlinearities through a series of multiple bodies, each composed of a linear finite element. The BHawC model of the SWT-2.3-108 blade used in the current study was initially curved in space and discretized into 16 linear elements. In other parts of the turbine where bearings are present, special elements are introduced and the drivetrain consists purely of torsional elements.

The aerodynamic force in $\mathrm{BHawC}$ is calculated at a given number of points (which varies depending on the turbine) on the blades positioned independently of the structural nodes. 


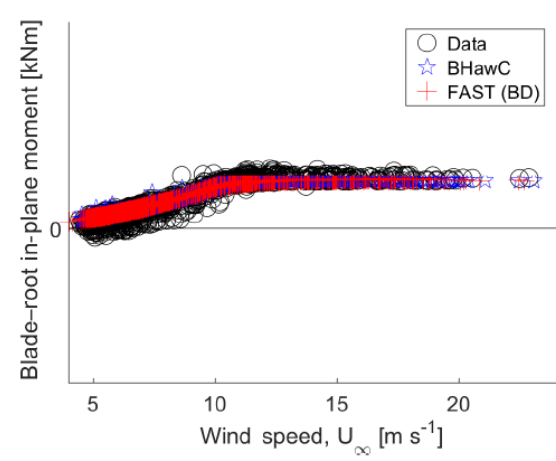

(a)

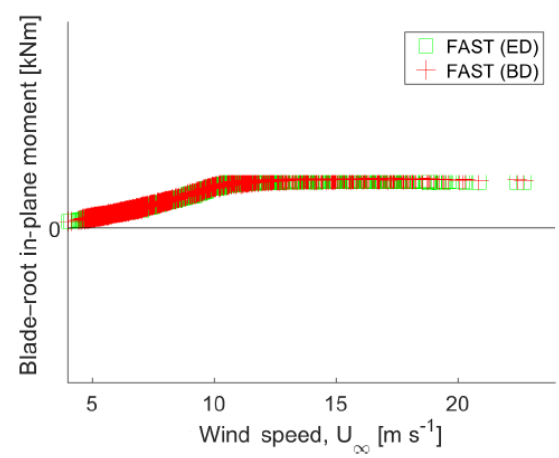

(c)

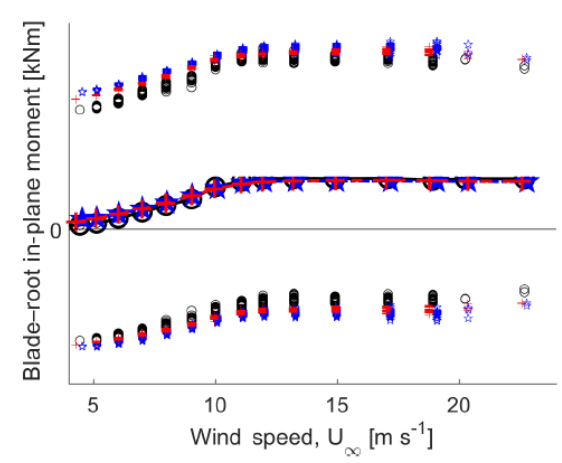

(b)

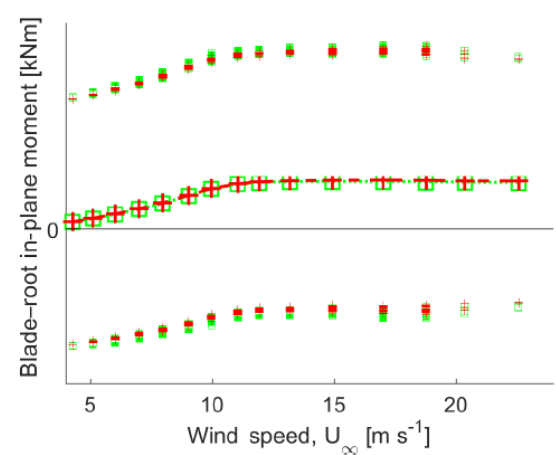

(d)

Figure 6. Blade-root in-plane bending moments. (a) Individual $10 \mathrm{~min}$ averages from experimental data, BHawC, and FAST (BD). (b) Binned averages of data in (a) are denoted by points connected by lines; the scatter above and below these data denotes plus or minus 1 standard deviation, respectively, of the individual $10 \mathrm{~min}$ data sets in that bin. (c) Individual 10 min averages from FAST (BD) and FAST (ED). (d) Binned averages of data in (c) are denoted by the points connected by lines; the scatter above and below these data denotes plus or minus 1 standard deviation, respectively, of the individual 10 min data sets in that bin.

Blade element momentum theory is applied to determine the tangentially and axially induced velocities at these aerodynamic calculation points, and Prandtl's tip loss correction as well as a correction for thrust at high induction values are implemented. The blade element implementation in BHawC also allows for unsteady and skewed inflow. The aerodynamic force is based on 3-D-corrected coefficients for stationary airfoil data, and a Beddoes-Leishman-type model for unsteady/dynamic events. In addition, BHawC contains a model for tower shadow, and it also calculates the aerodynamic forces on the nacelle and tower. For further details on BHawC, see (Skjoldan et al., 2011).

\subsection{Aeroelastic model of the SWT-2.3-108 machine}

The FAST model of the SWT-2.3-108 machine was created based on the information obtained from Siemens as well as the inflow data from the experimental database for each of the cases simulated. This three-bladed upwind turbine has a fixed coning angle of $5^{\circ}$ and a rotor tilt angle of $6^{\circ}$, both directing the blades away from the tower. The tower, blades, and the main shaft are modeled as elastic bodies with properties obtained from Siemens, and the tower base is fixed.
Data used for model calibration are generally recommended to be different from those used for validation (see, e.g., Bayarri et al., 2007), and accordingly, the information used to build the FAST model for the purpose of validation in this study has not been used for model calibration.

Details of the FAST v8 model have been described in a previous paper (Guntur et al., 2016). A part of this is reiterated below for thoroughness in the model description and also to provide the reader with the relative improvements since the last version of the turbine model.

In the BeamDyn, it is possible to model a blade defined by many cross-sectional property stations with relatively few node points for integration while capturing all of the provided material properties (Wang et al., 2016). The cross-sectional properties of the blades on the Siemens machine were defined at 106 stations along its length. For BeamDyn spectral finite-element calculations (e.g., those for mass and stiffness matrices), these data were spatially integrated using the trapezoidal rule, and each blade was discretized using a single seventh-order spectral finite element.

The two-dimensional airfoil aerodynamic properties for the blade were obtained from Siemens. The blade was modeled in AeroDyn using 20 nodes. The lift-coefficient data 


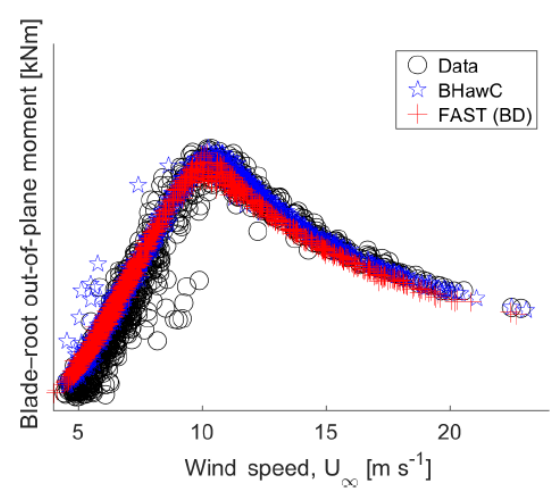

(a)

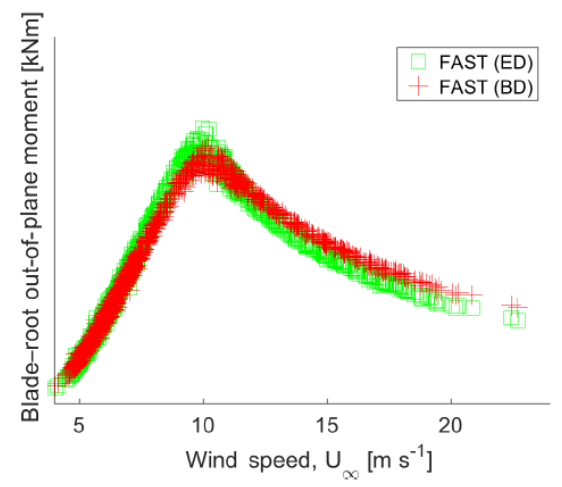

(c)

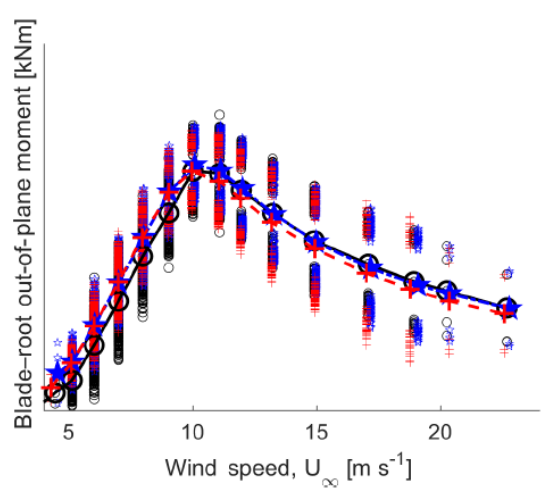

(b)

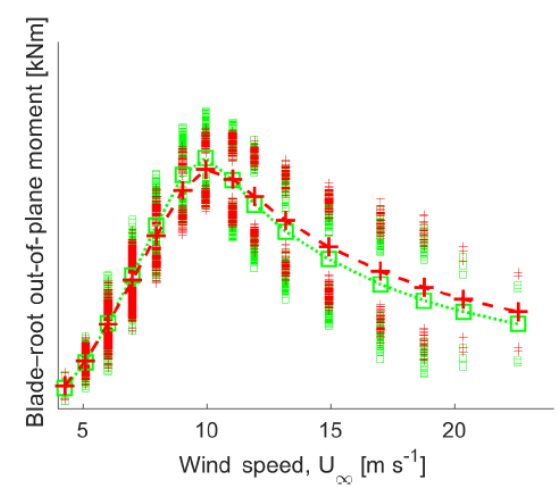

(d)

Figure 7. Blade-root out-of-rotor-plane bending moments. (a) Individual 10 min averages from experimental data, BHawC, and FAST (BD). (b) Binned averages of data in (a) are denoted by points connected by lines; the scatter above and below these data denotes plus or minus 1 standard deviation, respectively, of the individual 10 min data sets in that bin. (c) Individual 10 min averages from FAST (BD) and FAST (ED). (d) Binned averages of data in (c) are denoted by the points connected by lines; the scatter above and below these data denotes plus or minus 1 standard deviation, respectively, of the individual 10 min data sets in that bin.

were processed to include rotational augmentation effects in the same way as BHawC, according to a variation of the (Snel et al., 1993) model.

The measured yaw error in most test cases was very small, with a few exceptions of yaw errors higher than $5^{\circ}$ (based on a $10 \mathrm{~min}$ average of the wind-direction sensor readings obtained from the upwind met tower). Both BHawC and FAST simulations take this into account. In the FAST simulations, a fixed nacelle yaw error was used with a value equal to the 10 min average yaw error for each case.

FAST used the same turbulent inflow input files as BHawC, which used HawC-style turbulence boxes. For each case simulated, the turbulence box is scaled according to the mean wind speed and the turbulence intensity at the turbine hub height, obtained from the $80 \mathrm{~m}$ wind speed and direction measurement from the met tower.

A controller in a bladed-style dynamic-link library (DLL) form obtained from Siemens was used for pitch and torque control in the FAST simulations. It is known that this DLL controller does not employ the exact same control logic as the physical turbine or the $\mathrm{BHawC}$ simulations, but it is similar.

\section{Improvements to the FAST model of SWT-2.3-108}

The following are improvements to the current FAST model over the model used in the previous study (Guntur et al., 2016):

- In the FAST simulations in this paper, wind shear was modeled assuming a power-law profile. In the previous paper, a power-law exponent of 0.2 was assumed for all 1141 simulations, whereas in this work, the shear exponent was derived using experimental measurements. The met tower collected wind speed and direction information at the heights of $3,10,26,80,88$, and $134 \mathrm{~m}$. These data were used to estimate a power-law exponent by the least-squares best-fit method. For the few cases where a best-fit approximation was deemed unsuitable (e.g., low-level jet boundary layer profile), a power-law coefficient of 0.2 was assumed. The BHawC simulations used atmospheric shear estimates based on lidar measurements, and so there may be small differences among the inflow conditions of the test turbine, FAST simulations, and the $\mathrm{BHawC}$ simulations, adding to the uncertainty in this exercise. 


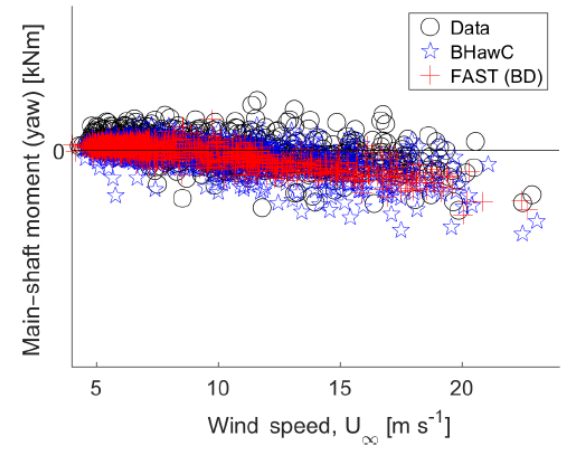

(a)

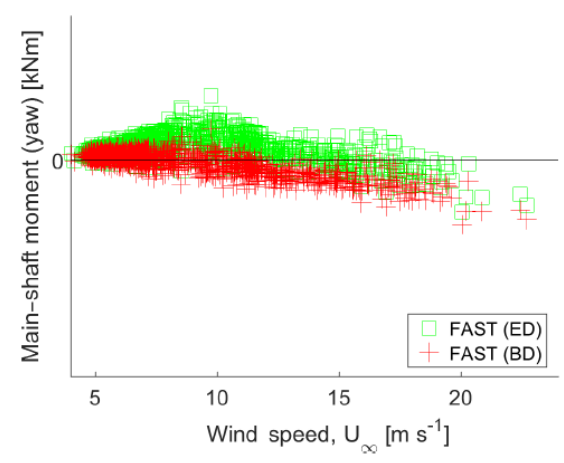

(c)

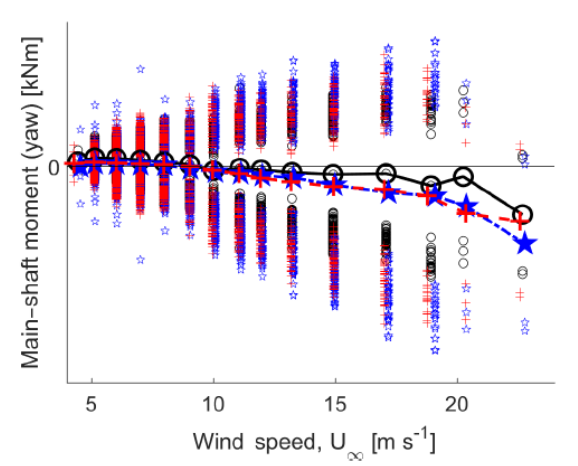

(b)

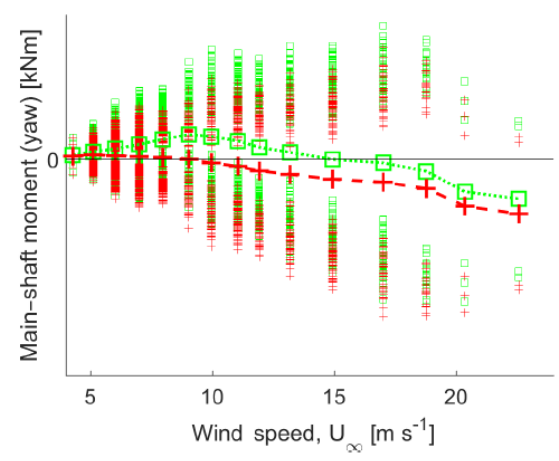

(d)

Figure 8. Main-shaft bending moment - yaw (nonrotating coordinate system). (a) Individual 10 min averages from experimental data, BHawC, and FAST (BD). (b) Binned averages of data in (a) are denoted by points connected by lines; the scatter above and below these data denotes plus or minus 1 standard deviation, respectively, of the individual 10 min data sets in that bin. (c) Individual 10 min averages from FAST (BD) and FAST (ED). (d) Binned averages of data in (c) are denoted by the points connected by lines; the scatter above and below these data denotes plus or minus 1 standard deviation, respectively, of the individual 10 min data sets in that bin.

- It was found that the rotor mass imbalance was modeled too high in the previous paper and has been corrected in the current model.

- A part of the experimental measurements of the mainshaft bending moments was found to contain corrupt data, and those data have been filtered out.

- A controller in a bladed-style DLL form obtained from Siemens was used for pitch and torque control in the FAST simulations. In the previous study, it was observed that the FAST simulations with the Siemens DLL controller gave rise to some resonance effects in the coupled drivetrain and tower side-side modes, which in the previous study were suppressed by artificially increasing the drivetrain damping. It was later found that the FAST-DLL controller interface requires a lowpass filter to filter out high-frequency content on the generator speed in order for the FAST model to represent the control mechanism as close to the BHawC model/experimental turbine as possible. This filter was added to FAST in the simulations shown in this paper, resolving the resonance issue highlighted earlier. The drivetrain properties used in the current simulations are those that were prescribed by Siemens (i.e., the damping was reduced to its original value in the new results); no further artificial changes have been made.

- The previous study did not include unsteady aerodynamics effects, which are included in this work (Ning et al., 2015; Damiani et al., 2016).

The simulations shown in this work were generated using FAST v8.15.01a-bjj compiled in double precision with

- ElastoDyn v1.03.02a-bjj,

- BeamDyn v1.01.01,

- AeroDyn v15.00.01a-bjj,

- InflowWind v3.02.00a-adp, and

- ServoDyn v1.04.00a-bjj.

The FAST simulations with BeamDyn used a time increment of $0.0005 \mathrm{~s}$, whereas those with ElastoDyn used $0.005 \mathrm{~s}$. All FAST simulations were carried out for $12 \mathrm{~min}$, where the first 2 min were ignored to remove initial-transient effects. BHawC simulations were $11 \mathrm{~min}$ long with a $0.02 \mathrm{~s}$ integration time increment, and the first minute of transience 


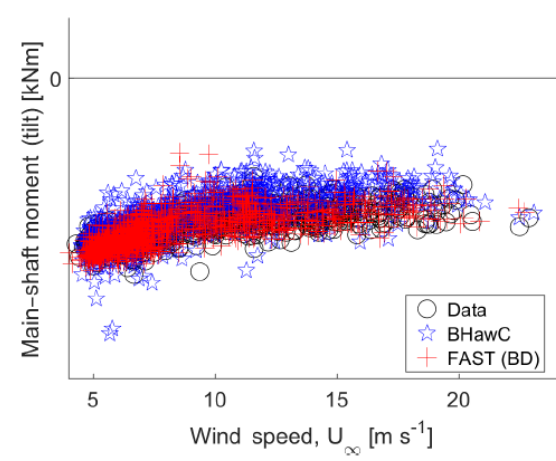

(a)

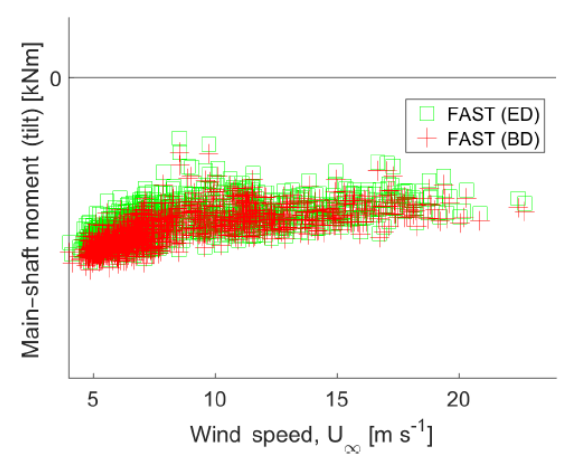

(c)

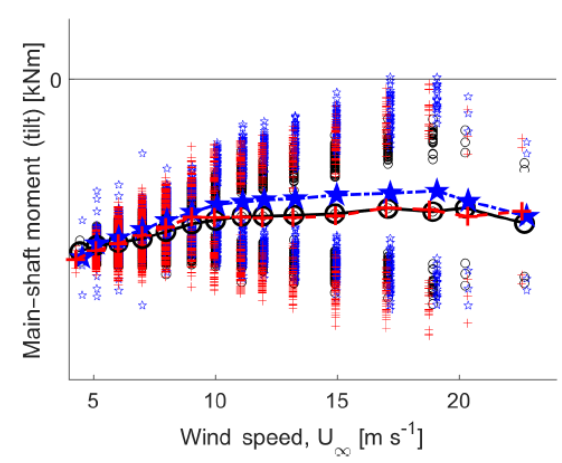

(b)

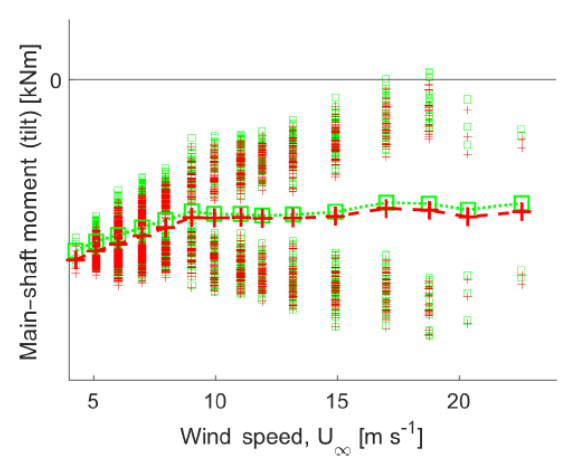

(d)

Figure 9. Main-shaft bending moment - tilt (nonrotating coordinate system). (a) Individual 10 min averages from experimental data, BHawC, and FAST (BD). (b) Binned averages of data in (a) are denoted by points connected by lines; the scatter above and below these data denotes plus or minus 1 standard deviation, respectively, of the individual $10 \mathrm{~min}$ data sets in that bin. (c) Individual 10 min averages from FAST (BD) and FAST (ED). (d) Binned averages of data in (c) are denoted by the points connected by lines; the scatter above and below these data denotes plus or minus 1 standard deviation, respectively, of the individual 10 min data sets in that bin.

was ignored. Note that the structural blade in ElastoDyn is straight and only includes bending DOFs, whereas the structural blade in BeamDyn is curved (as is the blade model in BHawC) and includes the DOFs bending, torsion, shear, and extension, with composite coupling terms.

\section{Results}

The plots presented herein show the mean and the standard deviation values of several QOIs for each of the $114110 \mathrm{~min}$ sample cases simulated, along with the PSDs of select bins at below-rated, at-rated, and above-rated operating conditions. The $y$ axes of the time-series plots as well as the $x$ axes of the frequency spectra plots have been removed to protect Siemens proprietary data, and so this paper presents a discussion based only on the relative trends among the different data shown. Each figure shows four different comparisons of the same QOI.

In the previous paper, each 10 min test case was simulated using three different FAST configurations, to be able to analyze the relative improvements in FAST among the capabilities in its previous public releases (FAST v8.10 and earlier) and the current developments with BeamDyn and AeroDyn.
One observation from the previous study has been that the effect of the aerodynamic blade curvature on the FAST results is negligible. Therefore, this study presents comparisons of FAST simulations with straight ElastoDyn blades and with curved BeamDyn blades, where both used curved aerodynamic blades. In the figures that follow, the different data identified in the legends are as follows:

- Data - experimental measurements

- BHawC - results from Siemens' BHawC simulations

- FAST (ED) - FAST simulations with ElastoDyn

- FAST (BD) - FAST simulations with the new BeamDyn.

Note that ElastoDyn and BeamDyn designate structural modules used to model the blades only; the structural dynamics of the remainder of the turbine (drivetrain, nacelle, and tower) were always modeled in ElastoDyn.

For Figs. 1 to 11, panels (a) and (b) show a comparison of the data from FAST with BeamDyn, BHawC, and experimental results, whereas panels (c) and (d) show those comparing FAST with BeamDyn with FAST with ElastoDyn. 


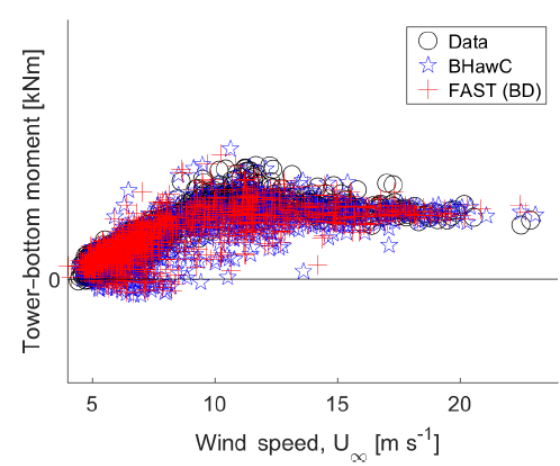

(a)

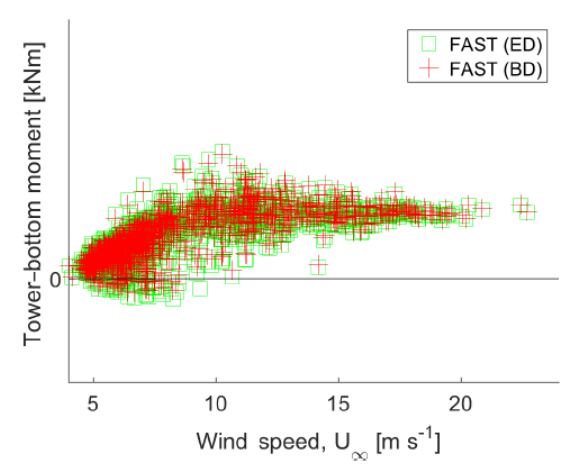

(c)

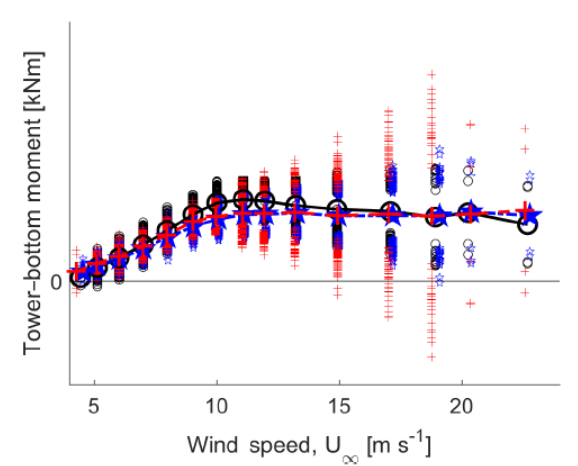

(b)

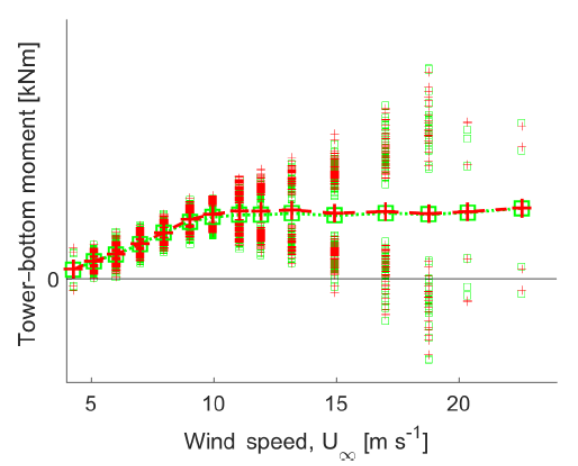

(d)

Figure 10. Tower-bottom side-side bending moments. (a) Individual 10 min averages from experimental data, BHawC, and FAST (BD). (b) Binned averages of data in (a) are denoted by points connected by lines; the scatter above and below these data denotes plus or minus 1 standard deviation, respectively, of the individual 10 min data sets in that bin. (c) Individual 10 min averages from FAST (BD) and FAST (ED). (d) Binned averages of data in (c) are denoted by the points connected by lines; the scatter above and below these data denotes plus or minus 1 standard deviation, respectively, of the individual 10 min data sets in that bin.

Panels (a) and (c) show the means of the individual $10 \mathrm{~min}$ data, while the panels (b) and (d) show the binned average means of the same QOIs and the standard deviations calculated from the individual $10 \mathrm{~min}$ data in a given bin. The data points connected by a line indicate the mean value of the binned data, calculated as the average of the means of the individual $10 \mathrm{~min}$ simulations within that bin. The scatter above and below each of the mean data shows 1 standard deviation of the individual 10 min data sets in that bin added and subtracted to the mean of that bin.

Figures 12 to 22 show the PSDs of three select bins to represent three hub-height wind speed bins: 8,12 , and $15 \mathrm{~m} \mathrm{~s}^{-1}$ (i.e., below-rated, rated, and above-rated wind speeds). Panels (a), (c), and (e) show comparisons of FAST with BeamDyn, BHawC, and experimental data, and panels (b), (d), and (f) show those of FAST with BeamDyn and FAST with ElastoDyn.

\subsection{Discussion of the results}

The current results consist of analyses in the time and frequency domains. The results indicate that the predictions from the latest version of FAST compare consistently well with those of BHawC as well as the experimental data. A comparison between the results from various FAST simulations indicate an improvement of the FAST simulations using BeamDyn over those using ElastoDyn.

The BHawC data shown in this paper are the same as those shown in (Guntur et al., 2016). The experimental data are also the same for all channels except the main-shaft bending moments, which have been filtered for some corrupt data that were identified. The FAST results shown here are generated using the FAST model with the new improvements discussed earlier in this paper.

The agreement among the results from the improved FAST model, BHawC, and experimental measurements remains very good. Below is a discussion regarding the observations from the comparisons presented in the figures and improvements to the results published in the previous study as a result of the newer FAST model.

\subsubsection{Comparisons using the means and the standard deviations}

Figures 1 and 2 show the electrical power and the rotor speed, respectively. Note that each point in the scatter shown in pan- 


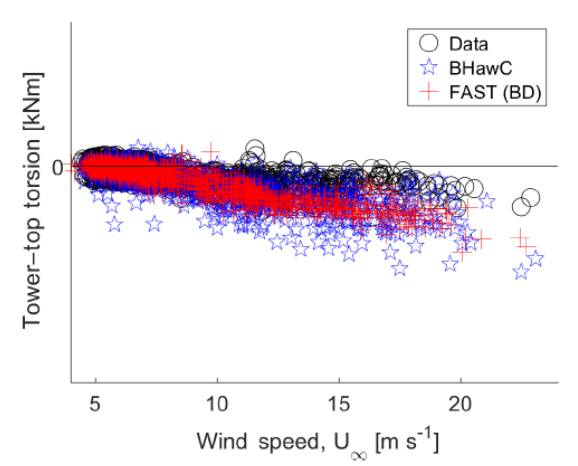

(a)

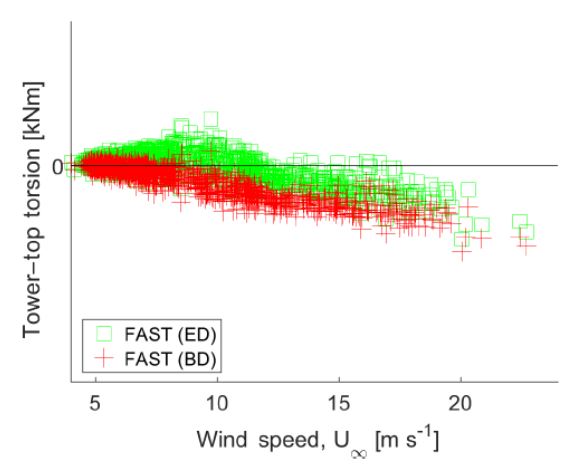

(c)

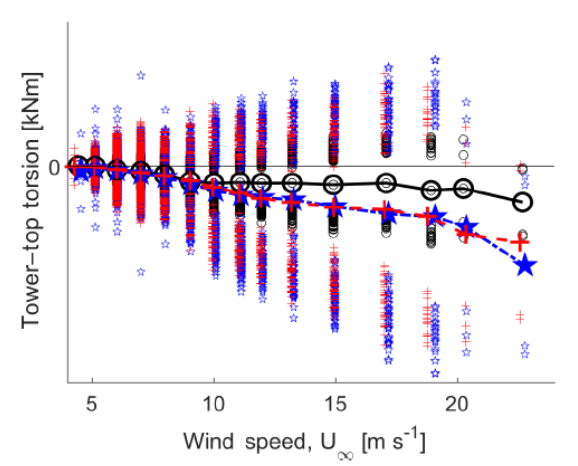

(b)

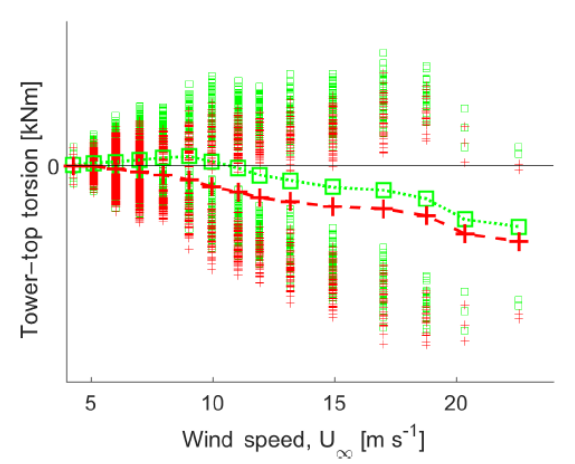

(d)

Figure 11. Tower-top torsion moment. (a) Individual 10 min averages from experimental data, BHawC, and FAST (BD). (b) Binned averages of data in (a) are denoted by points connected by lines; the scatter above and below these data denotes plus or minus 1 standard deviation, respectively, of the individual $10 \mathrm{~min}$ data sets in that bin. (c) Individual $10 \mathrm{~min}$ averages from FAST (BD) and FAST (ED). (d) Binned averages of data in (c) are denoted by the points connected by lines; the scatter above and below these data denotes plus or minus 1 standard deviation, respectively, of the individual $10 \mathrm{~min}$ data sets in that bin.

els (b) and (d) represents the standard deviation of the time series of an individual $10 \mathrm{~min}$ data set, added and subtracted to the mean of that bin. The agreement between the mean values of different data in electrical power is excellent. Figures 1a and 2a also show that there are some isolated cases in region 2 where the rotor speed and electrical power deviate from the general trend for this turbine, and it is worthwhile to note that these data have some influence on the variance in the load data presented next. These deviations are most likely due to a difference in the actual inflow field seen by the turbine compared to modeled turbulent inflow used in the aeroelastic models. Even so, the agreement in electrical power and the rotor speed between FAST, BHawC, and the experimental data is close enough to ensure that a majority of the turbine operating conditions being simulated and compared across FAST, BHawC, and the measurements are similar.

Figure 3 shows comparisons in the rotor thrust force. The rotor thrust is a difficult quantity to measure directly, and therefore, the measured thrust shown in this figure was estimated as the tower-bottom bending moment in the fore-aft direction divided by the tower height. Examining Figs. $3 \mathrm{~b}$ and $3 \mathrm{~d}$, it is evident that the BeamDyn model for the blade provides a better estimate of the thrust compared to Elas-
toDyn, particularly around rated conditions. At higher wind speeds, FAST simulations appear to show higher standard deviation values in the rotor thrust compared to those from measurements and BHawC results. On the other hand, the agreement between FAST and BHawC in the mean looks excellent, and the agreement between FAST and the experimental data looks reasonable.

Figures 4 and 5 show the in-plane and out-of-plane bladetip deflections from the simulations and the experimental measurements. The blade-tip deflection was not measured directly but was estimated using the fiber Bragg strain measurements from several locations along the span of Blade B. The local curvature was estimated from the strain measurements, which were then used to calculate the instantaneous shape of the blade. By integrating the shape, the magnitudes of the blade-tip deflections, in and out of the rotor plane, were obtained. From Figs. $4 \mathrm{c}$ and 5c, a significant difference between BeamDyn and ElastoDyn is visible. This difference is in fact an improvement, as revealed by Figs. 4a and 5a. The agreement of FAST with BeamDyn with the measurements as well as BHawC is very good throughout regions 2 and 3, and the agreement with the experimental data is particularly good in region 3. These figures show that BeamDyn is a significant 


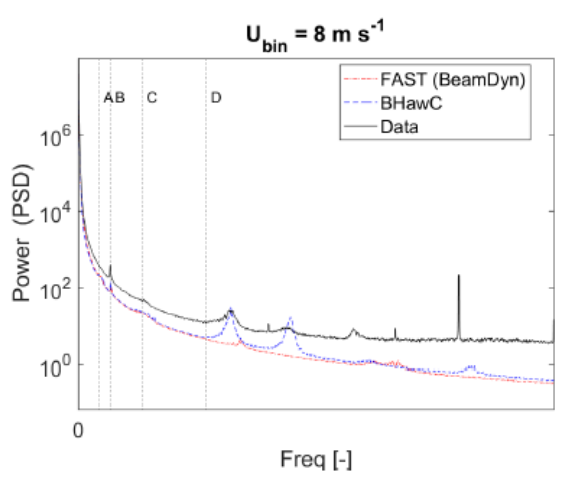

(a)

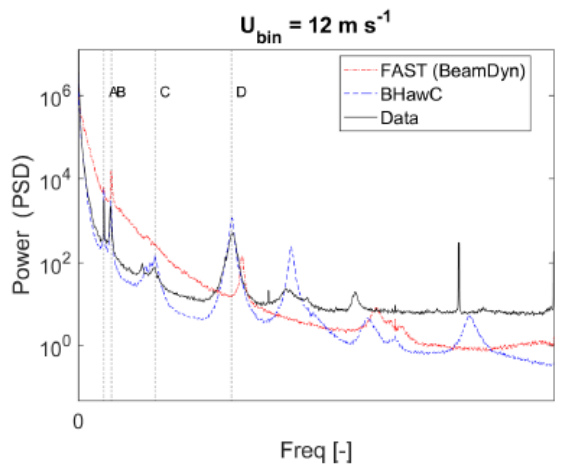

(c)

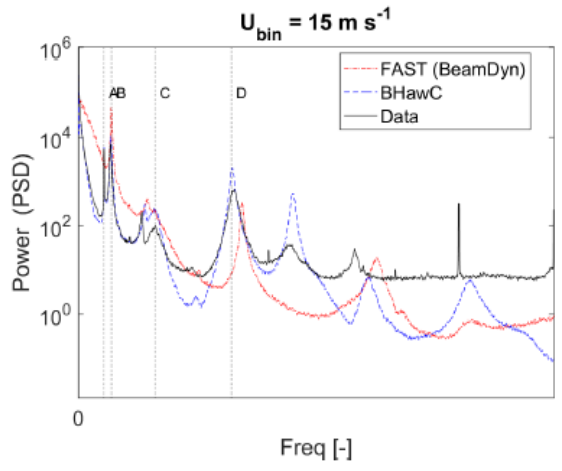

(e)

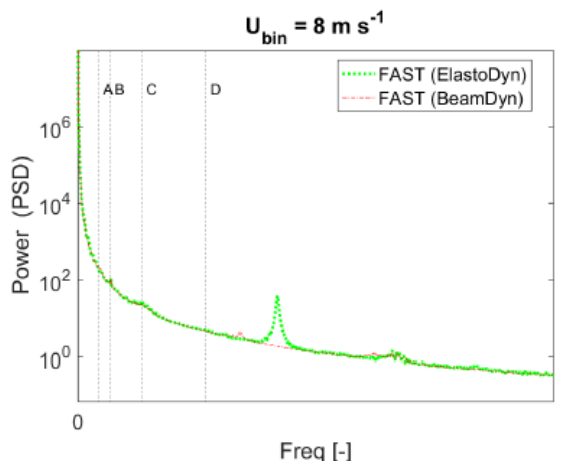

(b)

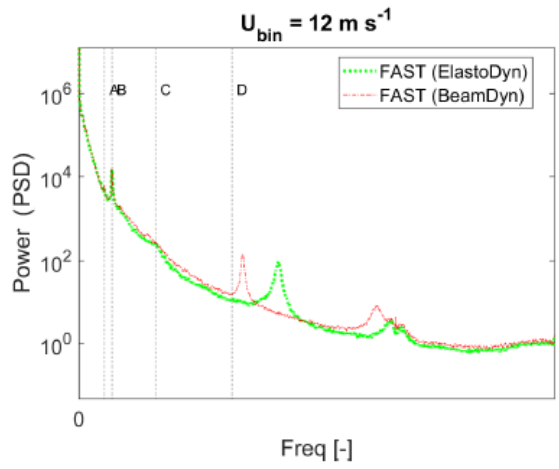

(d)

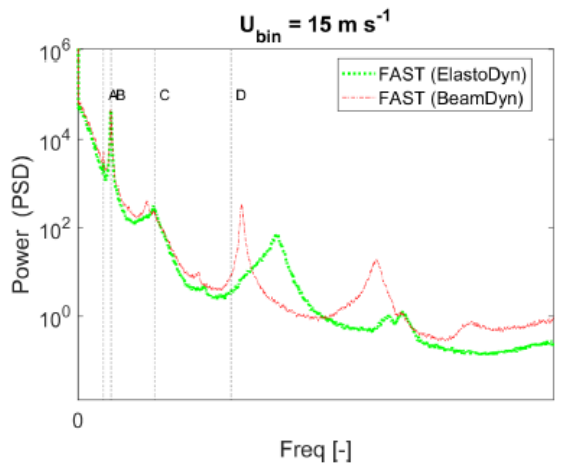

(f)

Figure 12. PSDs of the electrical power. The three sets of graphs represent the ensemble-averaged PSDs from the inflow velocity bins at $8 \mathrm{~m} \mathrm{~s}^{-1}$ (top), $12 \mathrm{~m} \mathrm{~s}^{-1}$ (center), and $15 \mathrm{~m} \mathrm{~s}^{-1}$ (bottom).

improvement over ElastoDyn. Note that only a fraction of the total set of experiments included reliable fiber Bragg data for example, the $8 \mathrm{~m} \mathrm{~s}^{-1}$ bin contained $10310 \mathrm{~min}$ data sets according to Table 1 , but only 21 of these contained usable fiber Bragg data. Because the PSDs of the blade-tip deflections shown in these figures were computed using fewer data sets, more noise is visible in these plots compared to other QOIs.

Figures 6 and 7 show the in-plane and out-of-plane bladeroot bending moments, respectively. Experimental data for the blade-root bending moments consist of strain-gage measurements at the roots of all three blades. For simplicity, only data from one of the three blades (Blade A) are being shown for the blade-root bending moments in this study. From the comparisons shown in Fig. 6a and b, fairly good agreement can be seen among the BHawC, experimental, and FAST (with BeamDyn) data. The in-plane blade-root bending moment is heavily sinusoidal, due to the weight of the blades (the amplitude of the oscillations is much higher than the mean, which is the reason why the standard deviations shown in Fig. $6 b$ and $d$ are so far away from the mean). From the out-of-plane blade-root bending moments shown in Fig. 7b and $7 \mathrm{~d}$, the overall agreement among FAST with BeamDyn, BHawC, and the measurements appears to be good. A small difference between the FAST results and the Siemens data is discernible, which may most likely be related to the small 


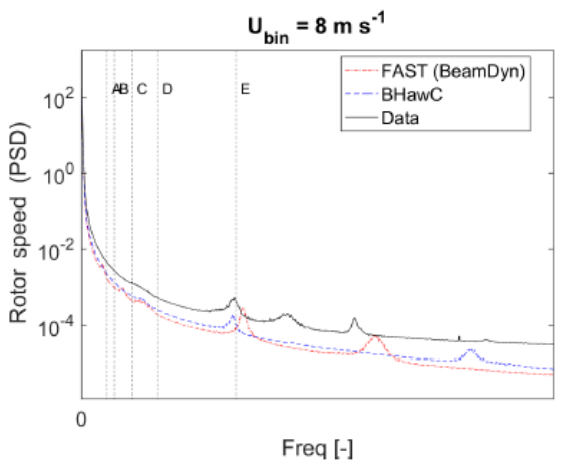

(a)

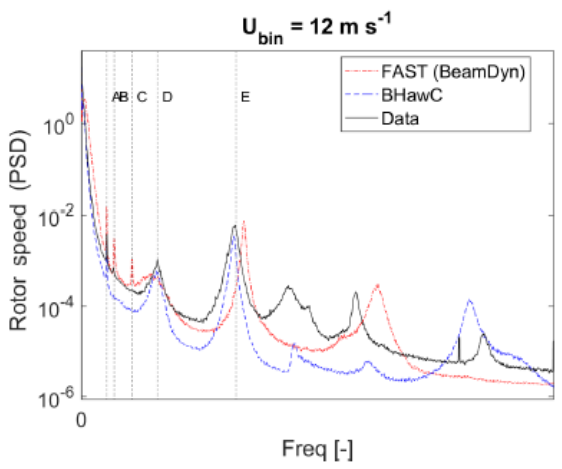

(c)

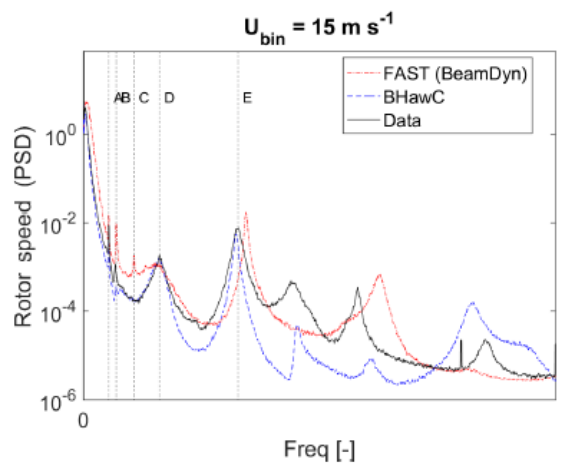

(e)

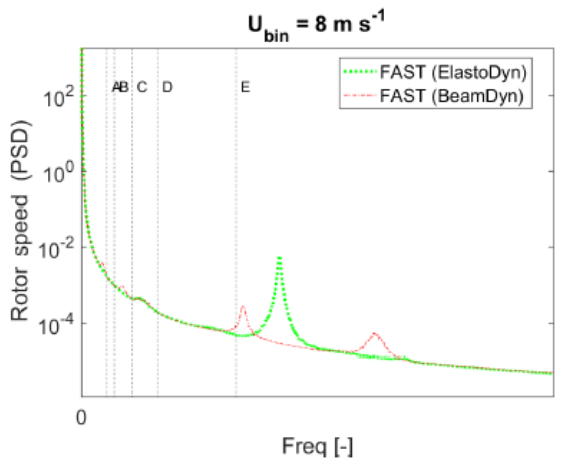

(b)

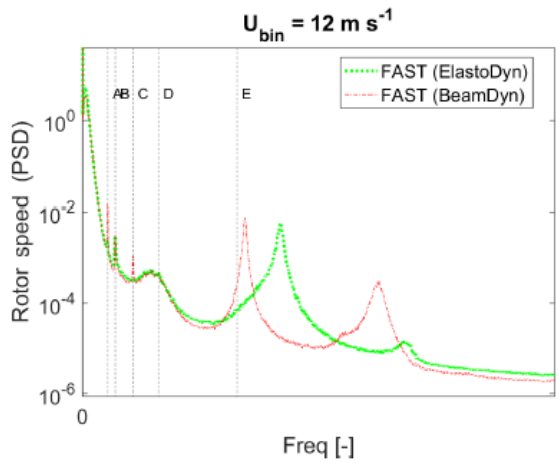

(d)

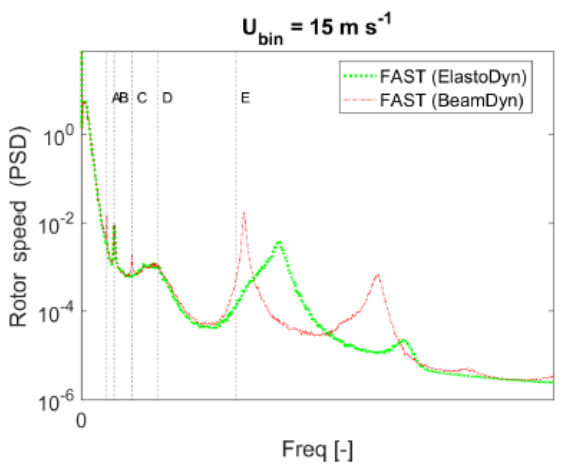

(f)

Figure 13. PSDs of the rotor speed. The three sets of graphs represent the ensemble-averaged PSDs from the inflow velocity bins at $8 \mathrm{~m} \mathrm{~s}{ }^{-1}$ (top), $12 \mathrm{~m} \mathrm{~s}^{-1}$ (center), and $15 \mathrm{~m} \mathrm{~s}^{-1}$ (bottom).

difference in the rotor speed also seen between FAST and Siemens data in Fig. 2, as discussed earlier. Furthermore, from Fig. 7c and d the improvement of FAST simulations with BeamDyn over ElastoDyn is clearly visible, more so at higher wind speeds.

The main-shaft yaw and tilt bending moments shown in Figs. 8 and 9 are strong functions of the drivetrain properties. As noted before, two improvements have been made to the experimental data shown in these figures: the experimental data have been processed to filter out corrupt data, and modifications to the controller and the drivetrain damping were made. As a result, results from FAST, BHawC, and the measurements show very good agreement in both the yaw and tilt directions. Particularly, FAST and BHawC results show excellent agreement in the mean as well as the standard deviations in the tilt direction, while the agreement between FAST and the experimental data is excellent in the yaw direction. As noted in the previous study, the moments in nonrotating coordinates are computed using the rotor azimuth and the moments in the rotating frame, and they therefore borrow from the uncertainties from the two quantities. Uncertainty quantification is outside of the scope of the current study, but it is useful to consider while comparing results from the codes to the experimental data.

Figure 10 shows the tower-bottom side-side (perpendicular to the prevailing wind direction) bending moments. An- 


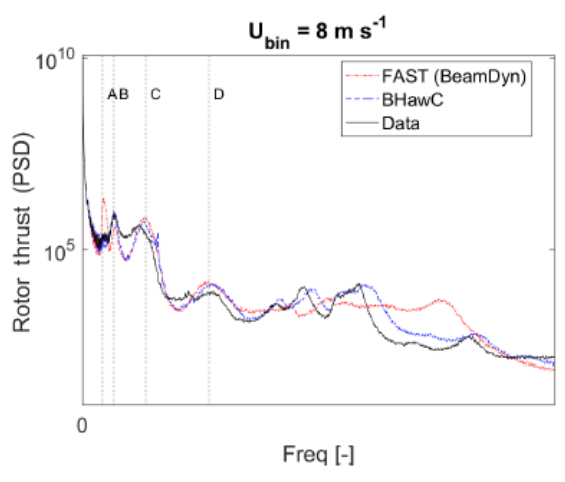

(a)

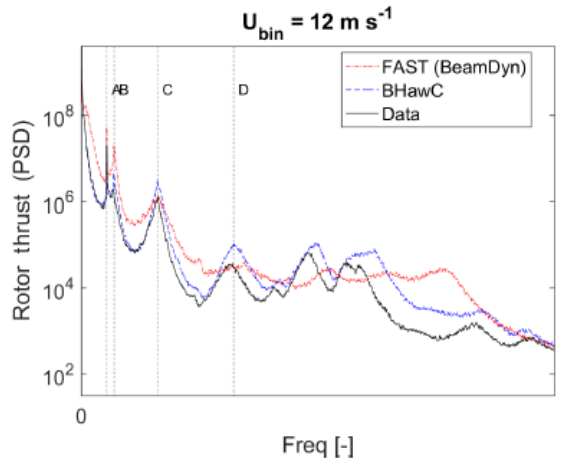

(c)

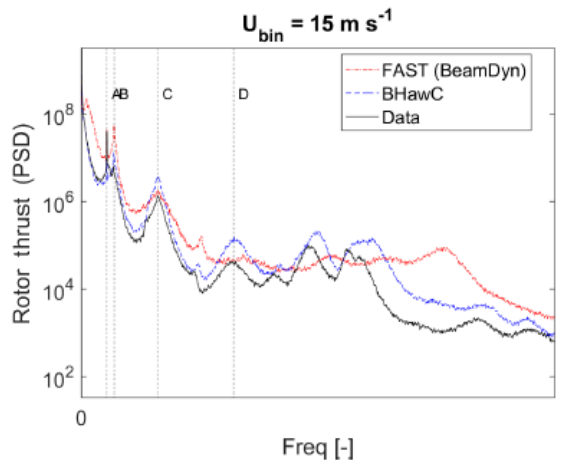

(e)

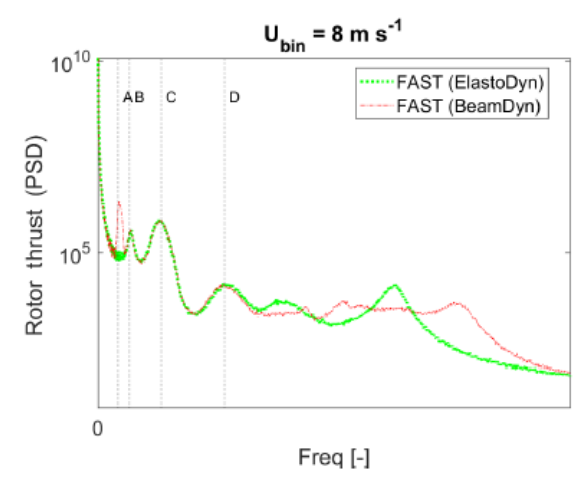

(b)

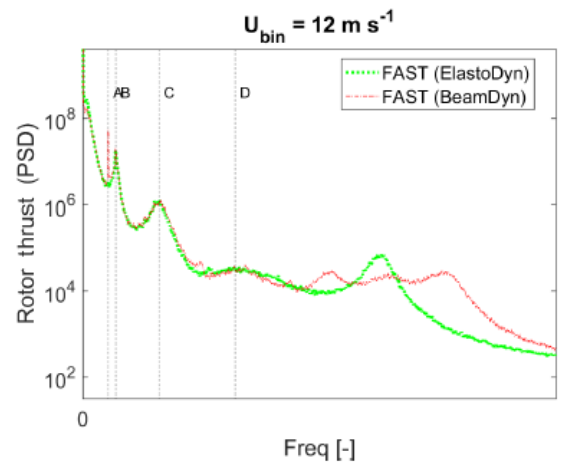

(d)

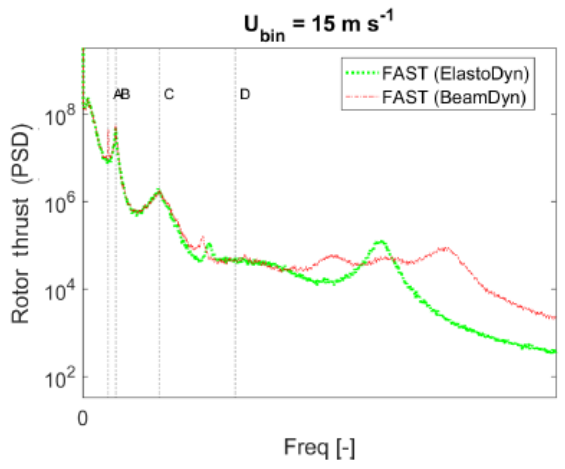

(f)

Figure 14. PSDs of the rotor thrust force. The three sets of graphs represent the ensemble-averaged PSDs from the inflow velocity bins at $8 \mathrm{~m} \mathrm{~s}^{-1}$ (top), $12 \mathrm{~m} \mathrm{~s}^{-1}$ (center), and $15 \mathrm{~m} \mathrm{~s}^{-1}$ (bottom).

other improvement to the FAST model mentioned earlier was the rotor mass imbalance that has been corrected in the model used in this paper. Improvements due to this correction can be observed in the tower-bottom side-side bending moments shown in Fig. 10.

Figure 11 shows the tower-top torsional moment. At lower wind speeds, the agreement between FAST with BeamDyn and the experimental data is very good. During at-rated and above-rated wind speeds, there seems to be good agreement between the FAST with BeamDyn and the BHawC results, but a deviation from the data is also shown. It is noteworthy to mention here that some uncertainty in the sensor calibration for this particular channel had been identified previously.
Once again, these measurements are sensitive to the vertical shear as well as the yaw error of the turbine, along with the difficulty associated with experimental measurement of this QOI, making a comparison of the mean values inherently challenging. Overall, the comparison between the numerical predictions of this QOI using FAST and BHawC shows good agreement.

\subsubsection{Discussion of results in the frequency domain}

Figures 12 to 22 show the PSDs of the different QOIs in three different bins, at below-rated, rated, and above-rated mean hub-height inflow wind speeds. Each PSD plot represents the 


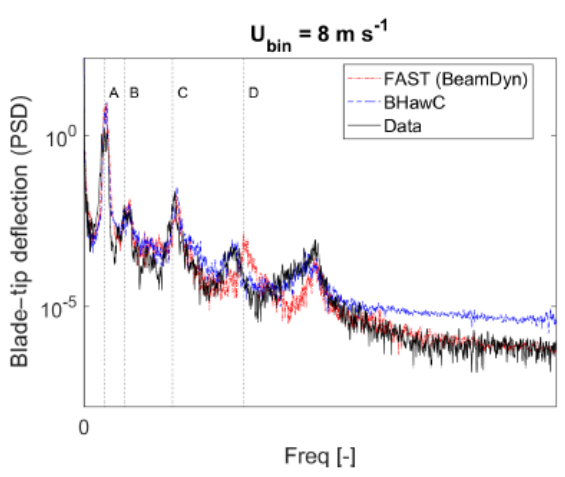

(a)

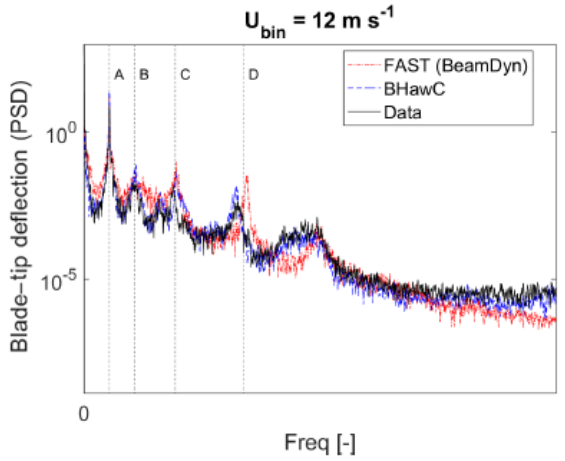

(c)

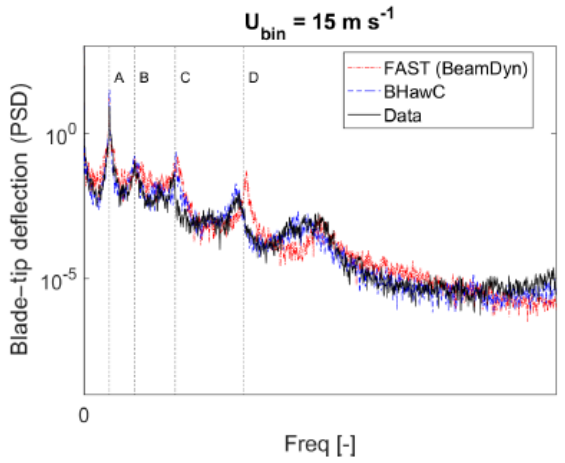

(e)

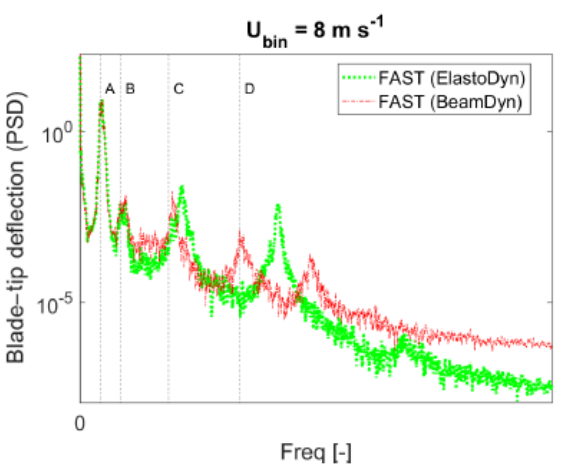

(b)

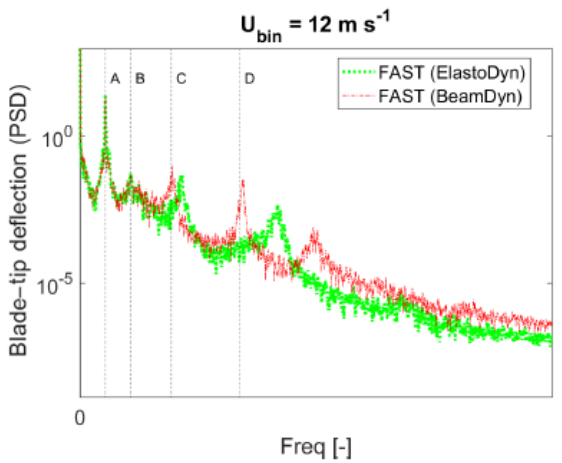

(d)

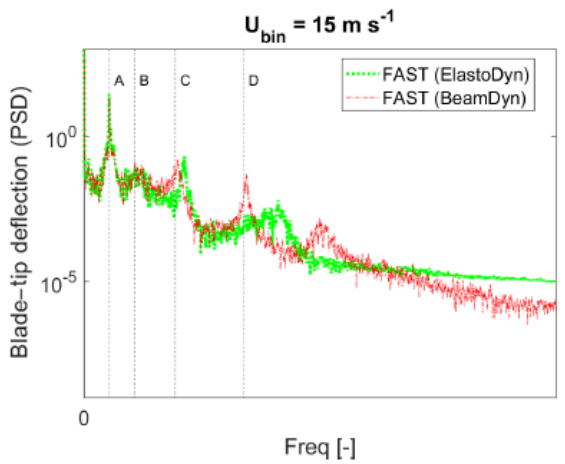

(f)

Figure 15. PSDs of the in-plane blade-tip deflection. The three sets of graphs represent the ensemble-averaged PSDs from the inflow velocity bins at $8 \mathrm{~m} \mathrm{~s}^{-1}$ (top), $12 \mathrm{~m} \mathrm{~s}^{-1}$ (center), and $15 \mathrm{~m} \mathrm{~s}^{-1}$ (bottom).

average of individual PSDs of the several 10 min data sets in the respective bin: for example, the PSD of a QOI at $8 \mathrm{~m} \mathrm{~s}^{-1}$ represents the average of 103 PSDs corresponding to the 103 individual data sets, according to Table 1. Each figure shows six PSD plots: the plots to the left (panels a, c, and e) show comparisons among FAST with BeamDyn, BHawC, and experimental measurements, and the plots to the right (panels $\mathrm{b}, \mathrm{d}$, and f) show comparisons between FAST with BeamDyn and FAST with ElastoDyn. The top two plots (panels a and b) represent the $8 \mathrm{~m} \mathrm{~s}^{-1}$ wind speed bin, which is below-rated operation. The two plots in the middle (panels $\mathrm{c}$ and d) represent the $12 \mathrm{~m} \mathrm{~s}^{-1}$ wind speed bin, which is approximately at-rated operation. The two plots at the bottom (panels e and f) represent the $15 \mathrm{~m} \mathrm{~s}^{-1}$ bin, which is above-rated operation. As highlighted before, the $x$ axes represent frequency and the values are hidden to protect Siemens data confidentiality. It has been highlighted in several previous studies that frequencies that are lower than approximately 10 times the rotor rated speed (1P) contribute most to fatigue loads (up to $2 \mathrm{~Hz}$ for turbines with $1 \mathrm{P}=0.2 \mathrm{~Hz}$; see Sim et al., 2012; Bergami and Gaunaa, 2014), and so the range shown in the figures in this paper includes the frequencies most important for load analysis. The peaks representing the frequencies with the highest energy in each figure have been highlighted and labeled as A, B, C, etc., and the comparisons among FAST, 


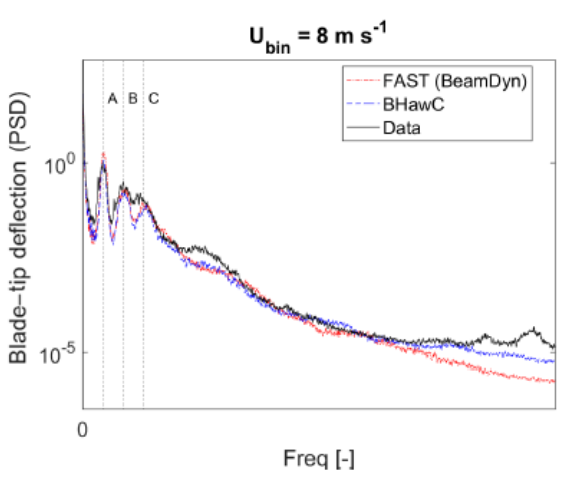

(a)

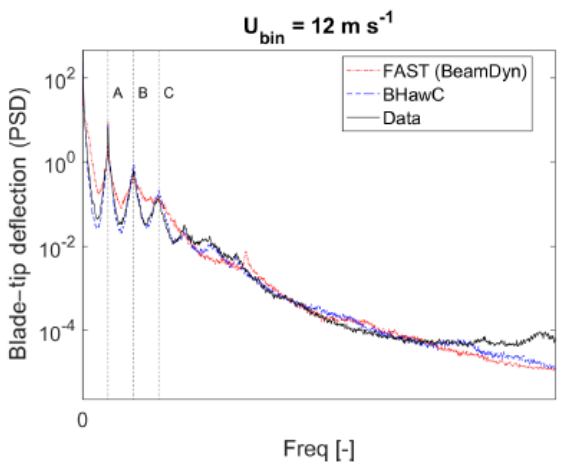

(c)

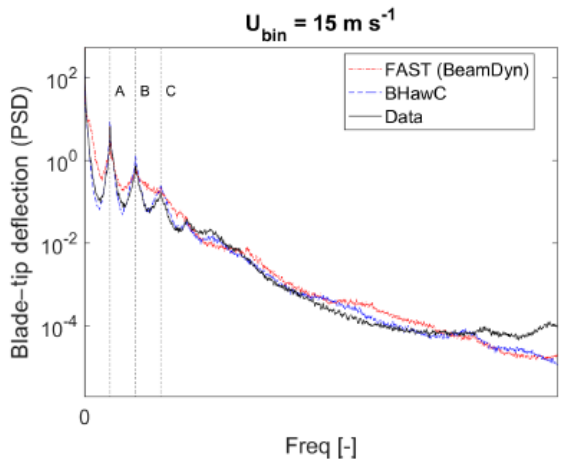

(e)

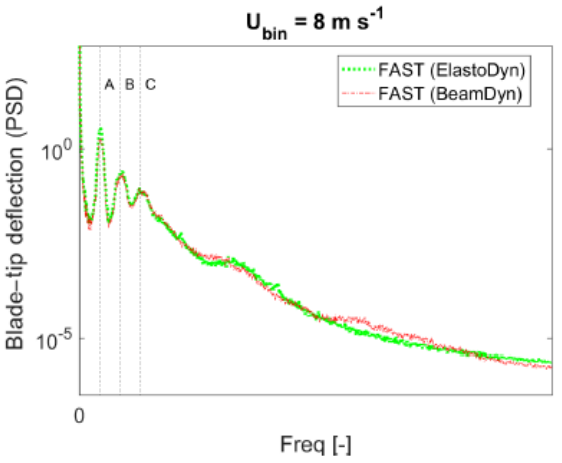

(b)

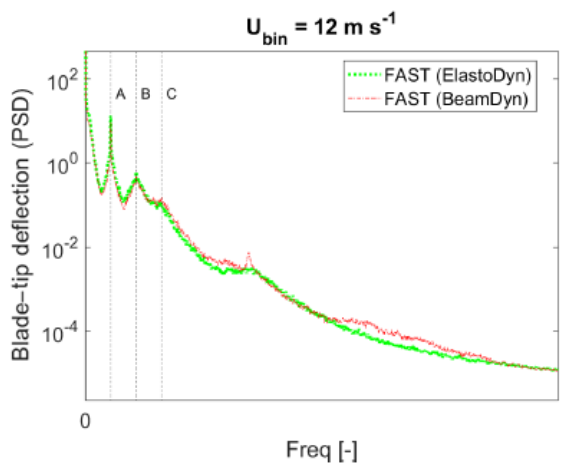

(d)

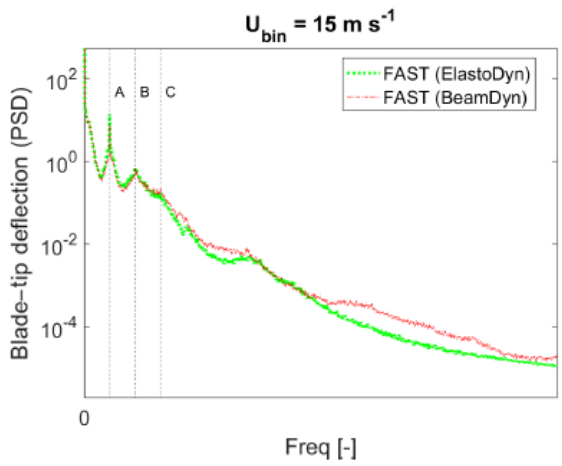

(f)

Figure 16. PSDs of the out-of-plane blade-tip deflection. The three sets of graphs represent the ensemble-averaged PSDs from the inflow velocity bins at $8 \mathrm{~m} \mathrm{~s}^{-1}$ (top), $12 \mathrm{~m} \mathrm{~s}^{-1}$ (center), and $15 \mathrm{~m} \mathrm{~s}^{-1}$ (bottom).

$\mathrm{BHawC}$, and the measurements presented in these figures are discussed relative to these peaks in the following paragraphs.

Figures 12 and 13 represent the PSDs of the electrical power and the rotor speed, respectively. Peaks $\mathrm{A}$ and $\mathrm{C}$ in Fig. 12 represent the rotor frequencies $1 \mathrm{P}$ and $3 \mathrm{P}$, respectively. Note that this rotor operates at $16 \mathrm{rpm}$ at rated power $(1 \mathrm{P} \simeq 0.267 \mathrm{~Hz})$. Peak $\mathrm{B}$ seems to be the tower side-side mode that couples with the rotor speed as well as the shaft torque, and peak $\mathrm{D}$ is most likely the drivetrain torsional mode. Peaks A, B, and $\mathrm{C}$ match quite well among FAST, BHawC, and the data. While FAST results are close to the $\mathrm{BHawC}$ results or the measurements, the small difference between them may be due to the difference in the drive- train models used. FAST results show higher energy in between the labeled peaks, particularly at lower frequencies and higher wind speeds. The reason for this is not certain - it may be a numerical artifact or due to the controller interface to FAST, but in any case it does not have a significant effect on either the dominant frequencies or the mean and the standard deviation values, as can be seen from Figs. 1 and 2.

Similarly, peaks A and D in Fig. 13 represent the rotor frequencies $1 \mathrm{P}$ and $3 \mathrm{P}$, respectively, peak $\mathrm{E}$ the drivetrain torsional mode, and peak B the tower side-side mode - all of which match quite well among FAST, BHawC, and the measurements. FAST results show some energy at peak $\mathrm{C}$ around the $2 \mathrm{P}$ frequency, which is not visible in the data or $\mathrm{BHawC}$ 


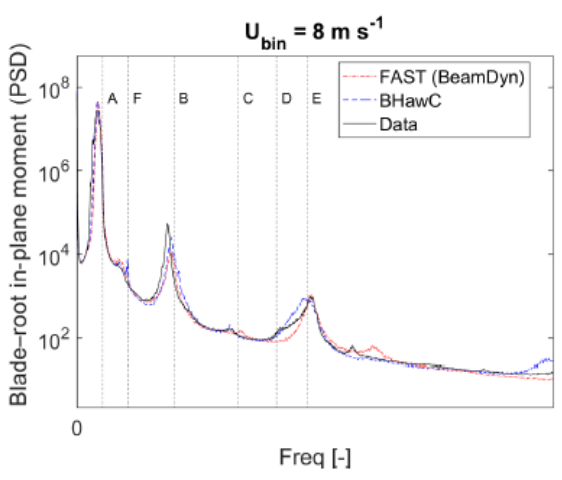

(a)

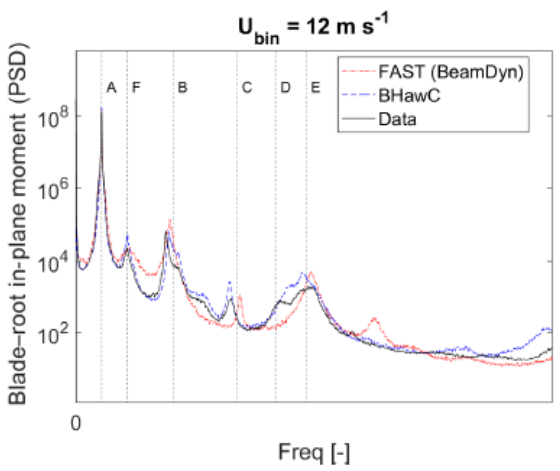

(c)

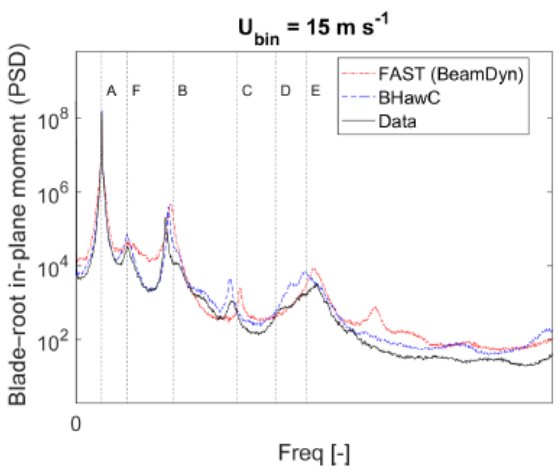

(e)

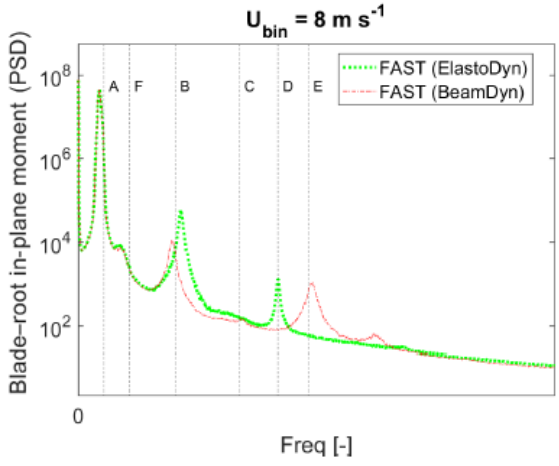

(b)

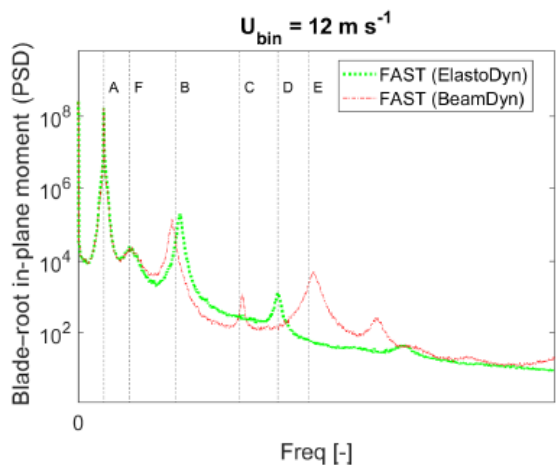

(d)

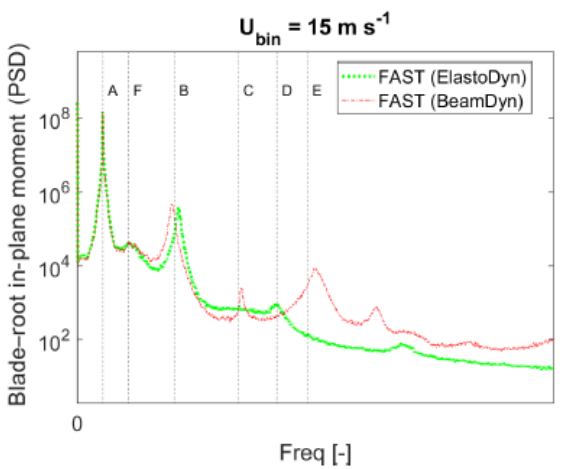

(f)

Figure 17. PSDs of the blade-root in-plane bending moments. The three sets of graphs represent the ensemble-averaged PSDs from the inflow velocity bins at $8 \mathrm{~m} \mathrm{~s}^{-1}$ (top), $12 \mathrm{~m} \mathrm{~s}^{-1}$ (center), and $15 \mathrm{~m} \mathrm{~s}^{-1}$ (bottom).

results. The exact reason for its presence in FAST and not the other channels is not certain, but considering the $y$ axis is a log scale, this peak has much less energy than the other dominant frequencies seen in the same plots.

PSDs of the rotor thrust force plotted in Fig. 14 also show good agreement between FAST and the data at the 1P and 3P frequencies denoted by peaks $\mathrm{A}$ and $\mathrm{C}$, respectively, as well as at peak B, which is the first tower fore-aft mode. Note that in the lower wind speed bin shown in Fig. 14a, the rotor speed varies depending on the instantaneous wind speed, which is why several peaks are noticed in the region immediately below $1 \mathrm{P}$.
The PSDs of the blade-tip deflections, shown in Figs. 15 and 16 , follow the trends observed in the blade-root bending moments. In Fig. 15, the peaks A and B denote $1 \mathrm{P}$ and $2 \mathrm{P}$, respectively; $\mathrm{C}$ represents the first blade-wise mode; and $\mathrm{D}$ represents the blade edge coupled with the drivetrain torsional mode. In Fig. 16, the peaks A, B, and C represent 1P, 2P, and 3P, respectively. The agreement among FAST with BeamDyn, BHawC, and the measurements is very good in both the in-plane as well as out-of-plane tip deflections. A comparison between ElastoDyn and BeamDyn results in Fig. 15b, d, and f reveals significant improvement in BeamDyn over ElastoDyn in accurately capturing the frequencies 


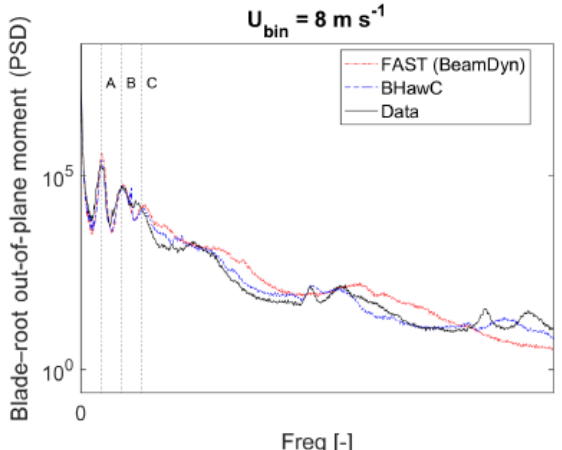

(a)

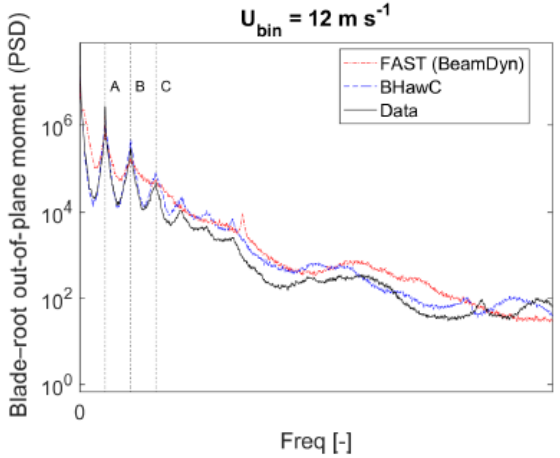

(c)

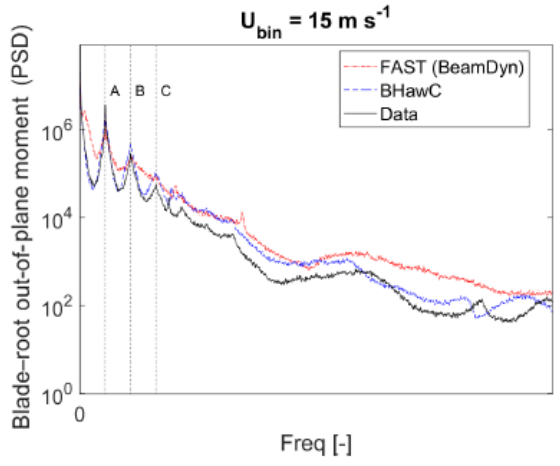

(e)

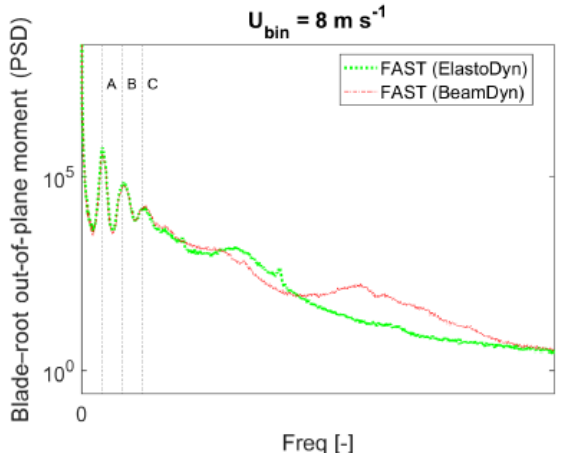

(b)

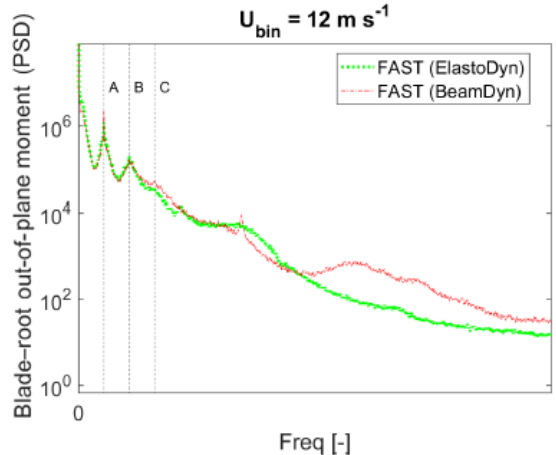

(d)

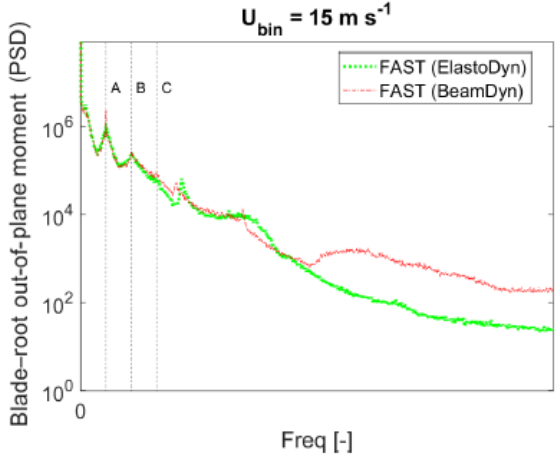

(f)

Figure 18. PSDs of the blade-root out-of-rotor-plane bending moments. The three sets of graphs represent the ensemble-averaged PSDs from the inflow velocity bins at $8 \mathrm{~m} \mathrm{~s}^{-1}$ (top), $12 \mathrm{~m} \mathrm{~s}^{-1}$ (center), and $15 \mathrm{~m} \mathrm{~s}^{-1}$ (bottom).

of those modes that are coupled with the blade edge - namely peaks $\mathrm{C}$ and $\mathrm{D}$.

The main difference between the two blade models used in the FAST simulations, ElastoDyn and BeamDyn, can most clearly be observed in the blade-root bending moments. The PSD of the blade-root in-plane bending moments shown in Fig. 17 shows two distinct features highlighting the improvements of using BeamDyn. Firstly, ElastoDyn simulations show the first blade-root in-plane bending moments at a higher value at peak $D$ rather than peak $C$ shown by BeamDyn. Secondly, the peak at E, which is most likely a coupled blade in-plane and torsional mode, are reproduced by BeamDyn simulations but not the model using ElastoDyn. Other peaks with the highest energies are peaks $\mathrm{A}$ and $\mathrm{F}$, which represent $1 \mathrm{P}$ and $2 \mathrm{P}$, respectively, and $\mathrm{B}$, which is most likely the first blade edge-wise mode. FAST with BeamDyn shows good agreement with the data for these modal frequencies during operation. There is a difference between FAST and the data outside the peaks, but this difference is ignored for the current analysis because, as mentioned before, the $y$ axes used here are on a log scale, and so these differences are very small compared to the energy at $1 \mathrm{P}$.

The PSDs of the blade-root out-of-plane bending moments are shown in Fig. 18. The peaks A, B, and C denote 1P, 2P, and $3 \mathrm{P}$, respectively. Because the frequencies in the out-ofplane direction for a tilted rotor are primarily dominated by 
Table 2. The tools FAST (BD), FAST (ED), and BHawC ranked according to how well their results compare to the experimental measurements in the mean and standard deviation for each QOI. No value indicates that no discernible difference could be seen among the different tools for that QOI.

\begin{tabular}{lcrc}
\hline QOI (time domain) & FAST (BD) & FAST (ED) & BHawC \\
\hline Elec. power & - & - & - \\
Rotor speed & 2 & 2 & 1 \\
Rotor thrust & 1 & 3 & 1 \\
Blade in-plane tip deflection & 1 & 3 & 2 \\
Blade out-of-plane tip deflection & 1 & 3 & 2 \\
Blade-root in-plane bending moment & - & - & - \\
Blade-root out-of-rotor-plane bending moment & 2 & 3 & 1 \\
Main-shaft bending moment - yaw & 1 & 3 & 1 \\
Main-shaft bending moment - tilt & 1 & 2 & 3 \\
Tower-bottom side-side bending moment & - & - & - \\
Tower-top torsion moment & 1 & 3 & 1 \\
\hline
\end{tabular}

Table 3. The tools FAST (BD), FAST (ED), and BHawC ranked according to how well their PSD results compare to the experimental measurements for each QOI. No value indicates that no discernible difference could be seen among the different tools for that QOI.

\begin{tabular}{lrrr}
\hline QOI (freq. domain) & FAST (BD) & FAST (ED) & BHawC \\
\hline Elec. power & 2 & 3 & 1 \\
Rotor speed & 2 & 3 & 1 \\
Rotor thrust & 2 & 2 & 1 \\
Blade-root in-plane bending moment & 1 & 3 & 1 \\
Blade-root out-of-rotor-plane bending moment & - & - & - \\
Blade in-plane tip deflection & 1 & 3 & 1 \\
Blade out-of-plane tip deflection & - & - & - \\
Main-shaft bending moment - yaw & 2 & 2 & 1 \\
Main-shaft bending moment - tilt & 2 & 2 & 1 \\
Tower-bottom side-side bending moment & 2 & 3 & 1 \\
Tower-top torsion moment & 2 & 2 & 1 \\
\hline
\end{tabular}

shear and gravity and because we also know that the rotor speeds match in all simulations, FAST (with ElastoDyn and BeamDyn), BHawC, and the measurements exhibit a similar behavior for this QOI.

Figures 19 and 20 show the PSDs of the main-shaft bending moments in the yaw and the tilt directions, respectively. The most dominant frequency at peak B matches well with the experiments and the data in both yaw and tilt directions, which is most likely the first shaft-torsion mode. The peak denoted by $\mathrm{C}$, which is most likely a blade-edge bending mode coupled to the shaft torsion, is visible and in agreement in the $8 \mathrm{~m} \mathrm{~s}^{-1}$ case among FAST, BHawC, and the measurements, while at higher wind speeds, the peak in the FAST data is not as conspicuous. Also, the 1P peak denoted by A is not as pronounced in the FAST simulations when compared to the data. These differences are most likely due to the combination of the filter used on the high-speed shaft in the FAST simulations to overcome the issues related to the controller, as mentioned earlier, and the limitations of the singletorsion-DOF drivetrain model used in FAST as opposed to a real drivetrain with multiple modes.
The PSDs of the tower-bottom side-side bending moments plotted in Fig. 21 show a good match of the 1P denoted by peak $A$, as well as the first tower side-side mode denoted by peak B. Plots $21 \mathrm{c}$ and $21 \mathrm{e}$ show a difference in the peak amplitude at peak $\mathrm{B}$. This reflects the difference in the standard deviation seen in Fig. 10 between FAST and the measurements. As seen, the frequency of this mode is accurately captured by FAST, but this mode seems to be less damped than in BHawC or the measurements, which may be related to the tuning of the DLL controller. The PSD of the towertop torsional moments in Fig. 22 also shows good agreement among FAST, the data, and $\mathrm{BHawC}$ results at the most prominent peaks in $\mathrm{A}$ and $\mathrm{B}$. Higher torsional modes, such as peak $\mathrm{C}$, shown by BHawC and the measurements, are not modeled by ElastoDyn.

\section{Conclusions}

In this paper, the latest results from FAST v8 with the new BeamDyn and AeroDyn modules have been presented. The release of BeamDyn and the AeroDyn overhaul within FAST 


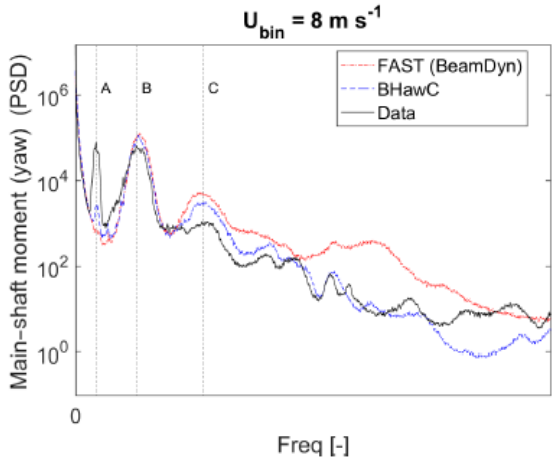

(a)

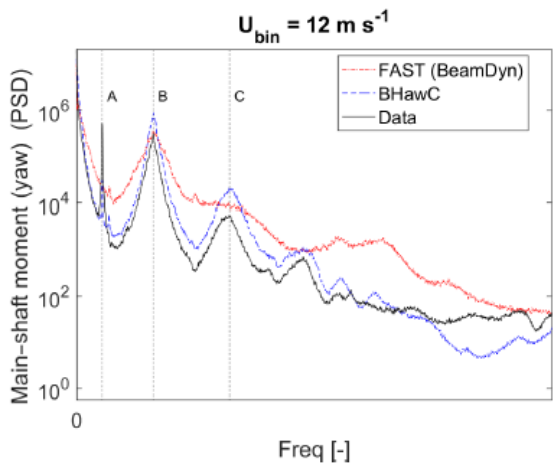

(c)

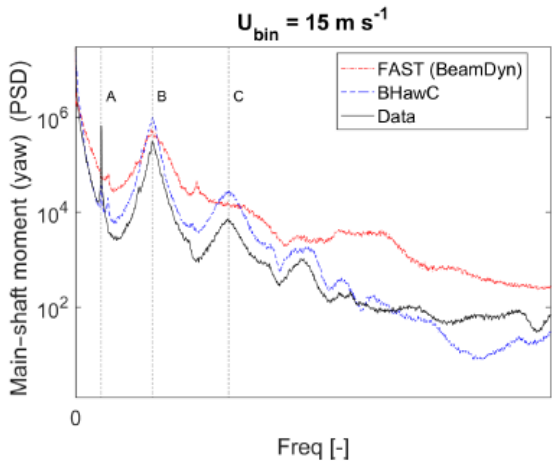

(e)

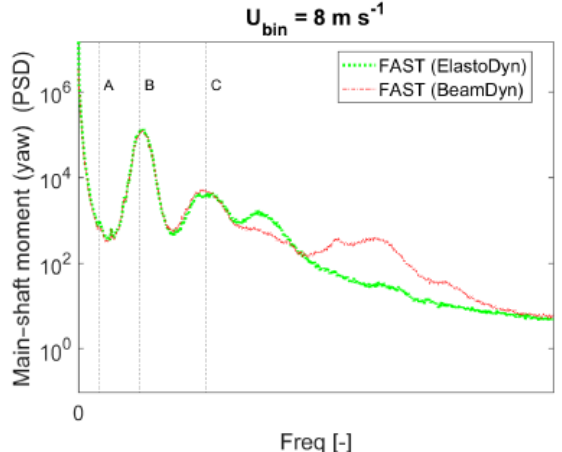

(b)

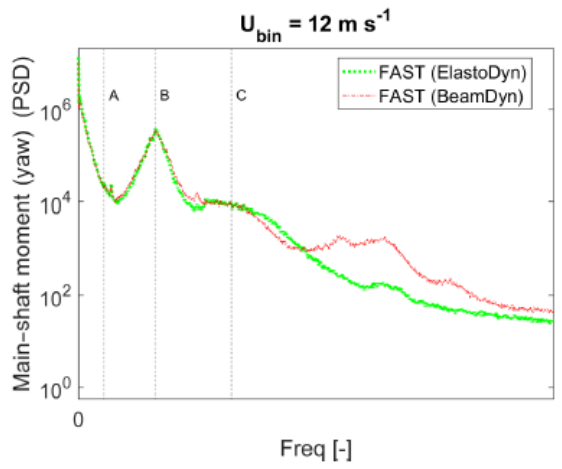

(d)

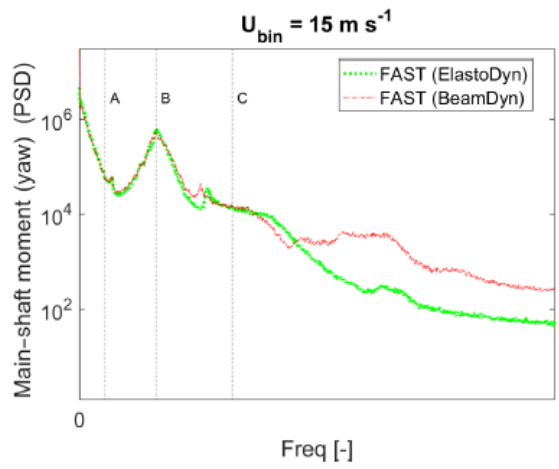

(f)

Figure 19. PSDs of the main-shaft bending moment - yaw (nonrotating coordinate system). The three sets of graphs represent the ensembleaveraged PSDs from the inflow velocity bins at $8 \mathrm{~m} \mathrm{~s}^{-1}$ (top), $12 \mathrm{~m} \mathrm{~s}^{-1}$ (center), and $15 \mathrm{~m} \mathrm{~s}^{-1}$ (bottom).

v8 opens up new possibilities in modeling and designing advanced aeroelastically tailored blades. Following the IEC61400-13 standard as a guideline, a comparison has been presented between FAST with and without its latest improvements, Siemens' in-house code BHawC, and the experimental data that were acquired from a series of field-test measurements from the Siemens 2.3 MW wind turbine at the NWTC.

Tables 2 and 3 show the tools FAST with BeamDyn, FAST with ElastoDyn, and BHawC, ranked according to how well their results compare qualitatively with the experimental measurements - the lower the rank, the better the agreement with the measured data. Note that this ranking is purely qualitative and does not take into account any measurement uncertainties in the experimental data. Results reveal significant improvements in the modeling capabilities of the latest version of FAST over its previous capabilities in most of the QOIs investigated in this paper. From Table 2, FAST with BeamDyn shows excellent agreement with the measurements in the time domain in general. This agreement is significantly closer to the measurements than ElastoDyn simulations and on par with and in some cases marginally closer to the measurements than BHawC. From Table 3, FAST with BeamDyn is shown to consistently deliver more accurate predictions compared to ElastoDyn. The FAST simulations with BeamDyn do not compare as well with the experimental data 


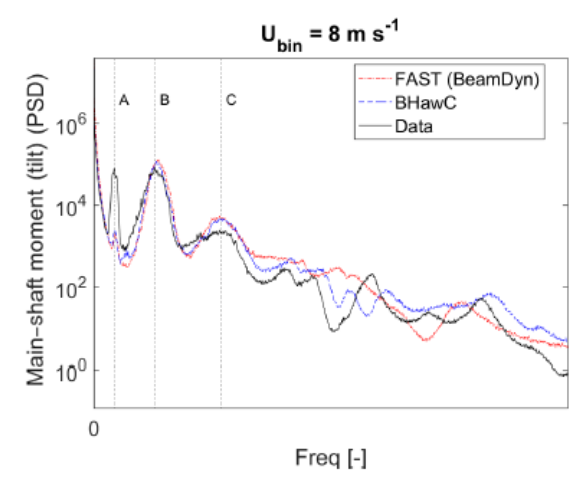

(a)

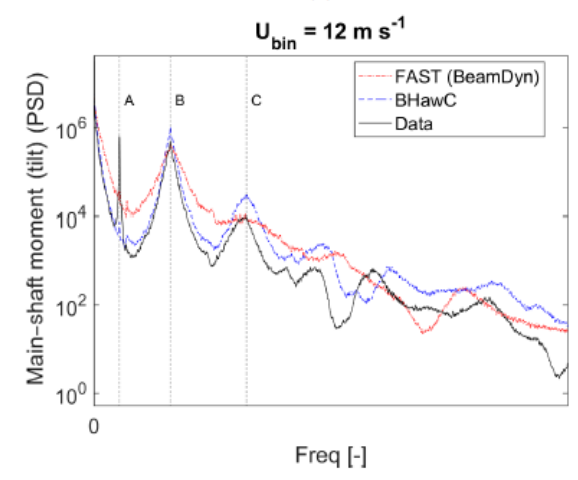

(c)

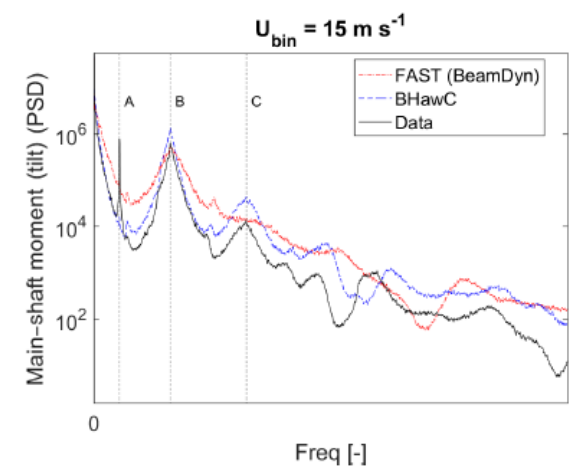

(e)

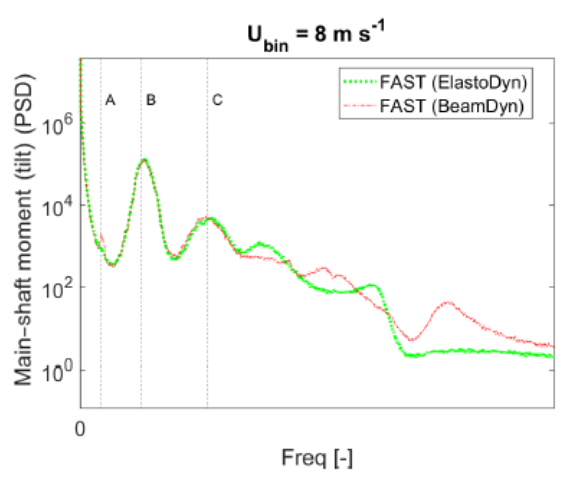

(b)

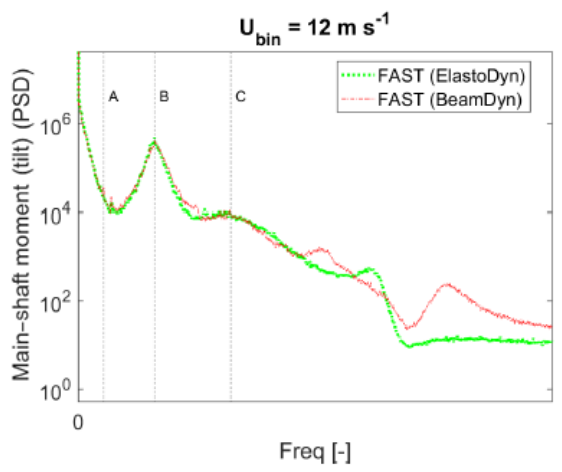

(d)

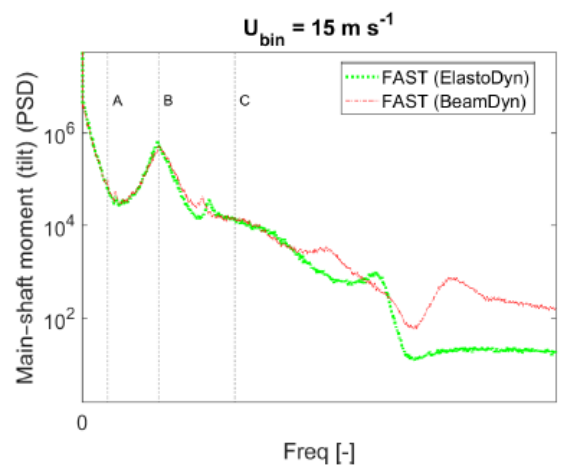

(f)

Figure 20. PSDs of the main-shaft bending moment - tilt (nonrotating coordinate system). The three sets of graphs represent the ensembleaveraged PSDs from the inflow velocity bins at $8 \mathrm{~m} \mathrm{~s}^{-1}$ (top), $12 \mathrm{~m} \mathrm{~s}^{-1}$ (center), and $15 \mathrm{~m} \mathrm{~s}^{-1}$ (bottom).

as $\mathrm{BHawC}$, but this is most likely because of the difference in the controller and the drivetrain models.

Based on the presented results overall, FAST v8 has been sufficiently validated against field measurements, along with a code-to-code verification with BHawC. FAST $\mathrm{v} 8$ has been demonstrated to be valid for aeroelastic load analyses of wind turbines, even with blades with significant aeroelastic tailoring.

The results did not include uncertainties that are inherent to the experimental data-acquisition process as well as the modeling and simulation process. For example, there may be errors due to the differences in the controller used in the simulation compared to the actual machine; there may be uncer- tainties associated with the structural properties of the blades, tower, drivetrain, etc.; there may be uncertainties due to the limitations in the information available about the inflow wind and shear conditions measured that were used to recreate the turbulence boxes; and there may be a measurement error that has not been quantified in this paper. Analysis of all such uncertainties and their impact on the results requires a detailed, separate work, which will be a part of future work. In addition, validation in conditions with elevated yaw errors and wind speed variations, both associated with extreme conditions, correspond to disparate physics and numerics and require special considerations, which will also be a part of future work. 


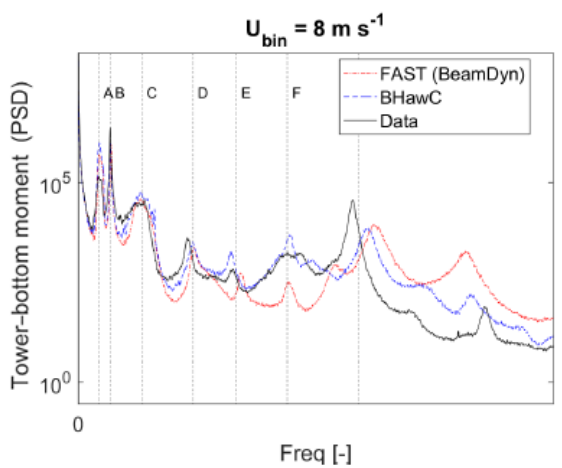

(a)

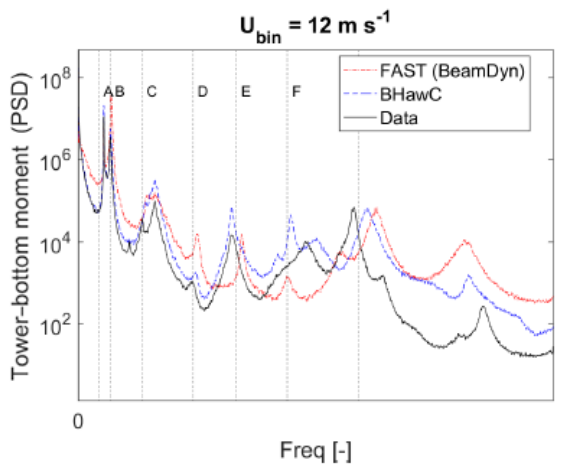

(c)

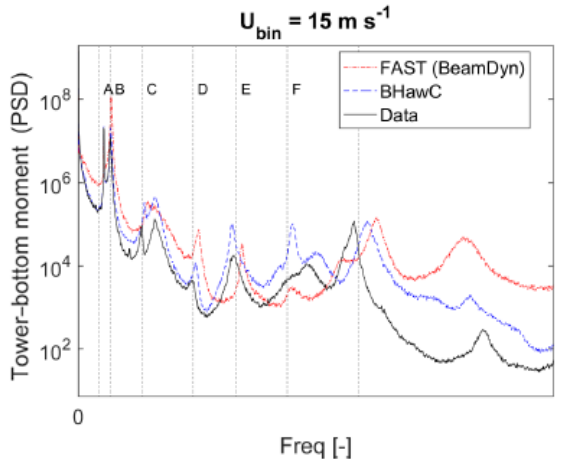

(e)

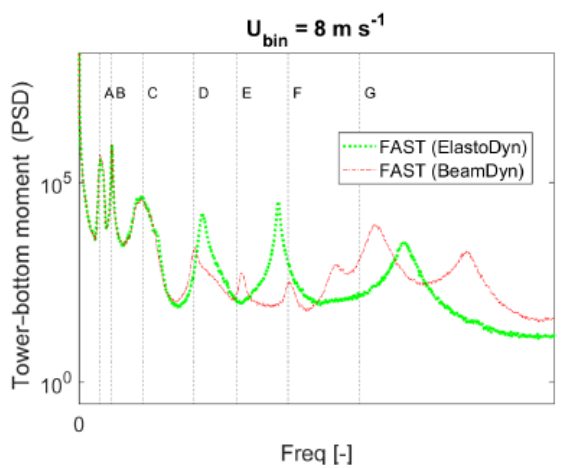

(b)

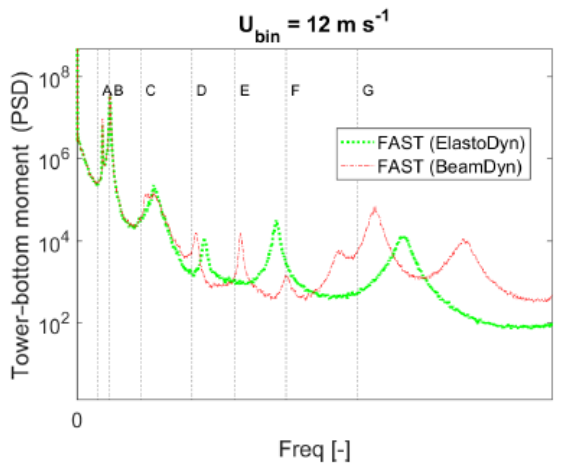

(d)

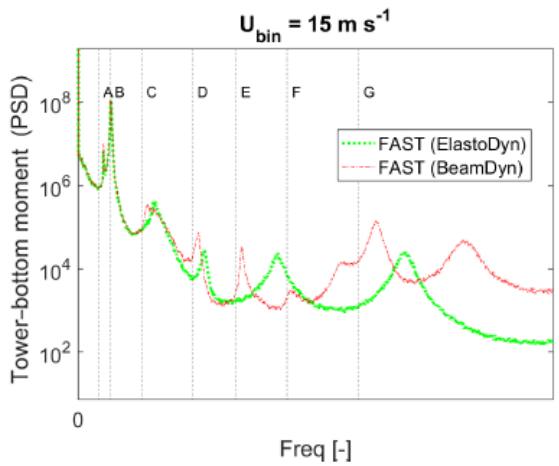

(f)

Figure 21. PSDs of the tower-bottom side-side bending moments. The three sets of graphs represent the ensemble-averaged PSDs from the inflow velocity bins at $8 \mathrm{~m} \mathrm{~s}^{-1}$ (top), $12 \mathrm{~m} \mathrm{~s}^{-1}$ (center), and $15 \mathrm{~m} \mathrm{~s}^{-1}$ (bottom). 


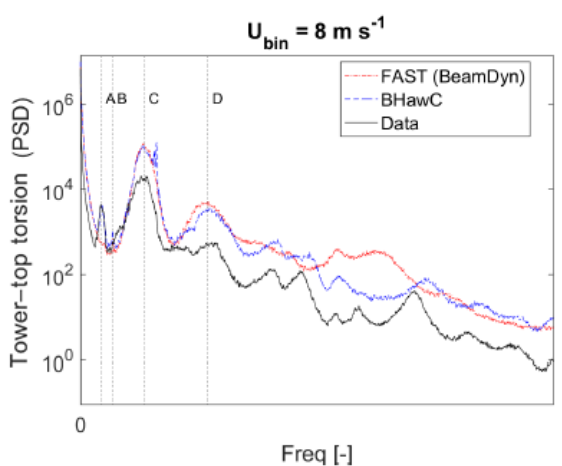

(a)

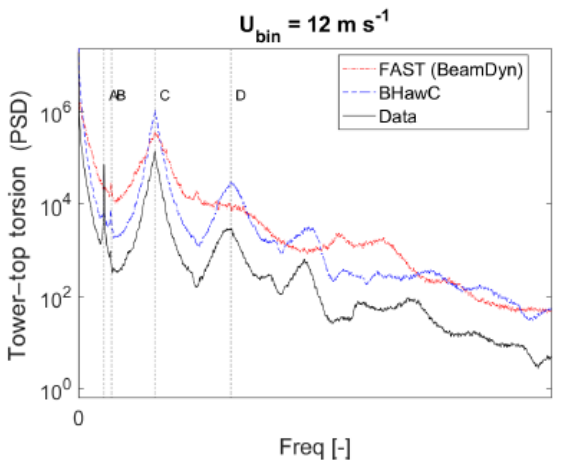

(c)

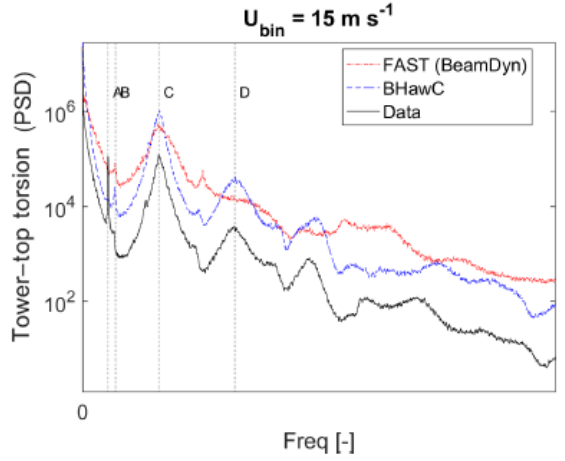

(e)

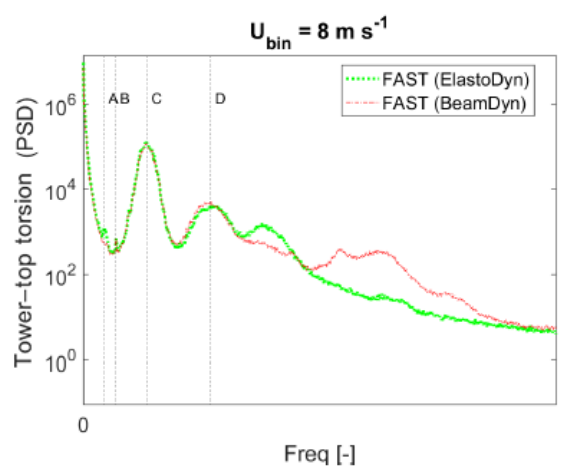

(b)

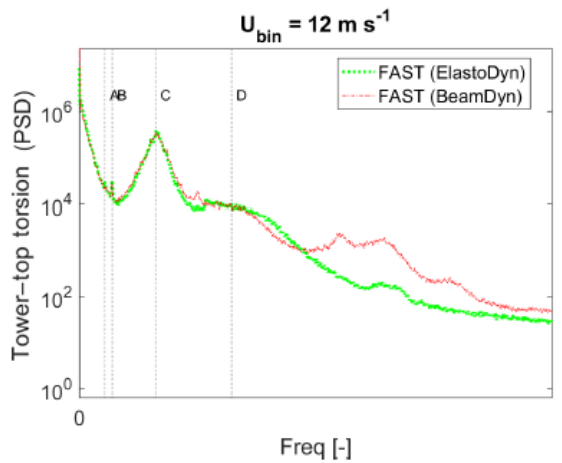

(d)

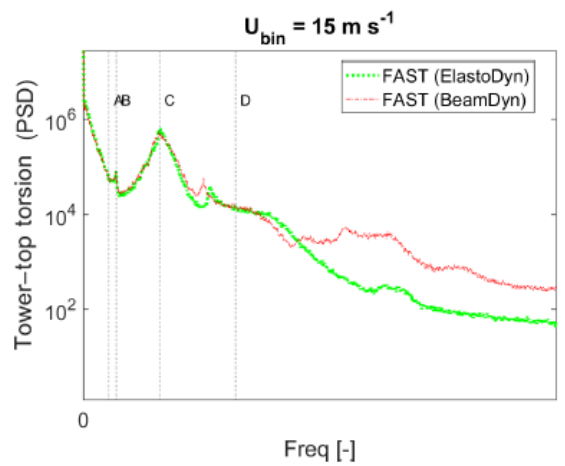

(f)

Figure 22. PSDs of the tower-top torsion moment. The three sets of graphs represent the ensemble-averaged PSDs from the inflow velocity bins at $8 \mathrm{~m} \mathrm{~s}^{-1}$ (top), $12 \mathrm{~m} \mathrm{~s}^{-1}$ (center), and $15 \mathrm{~m} \mathrm{~s}^{-1}$ (bottom). 
Code availability. The software package FAST v8, the source code, its submodules, and related information can be accessed for free at https://nwtc.nrel.gov/FAST (NWTC Information Portal (FAST), 2015). The data concerning the turbine is Siemens proprietary information and were shared with NREL under a nondisclosure agreement (NDA). Interested readers may contact the corresponding author for more information.

Competing interests. Srinivas Guntur, Jason Jonkman, Michael A. Sprague, Scott Schreck, and Qi Wang are affiliated with NREL, which produced the FAST tool. Ryan Sievers is employed by Siemens, who produced the turbines from which data were obtained and who produced BHawC.

Acknowledgements. This work is the outcome of a collaborative research and development agreement between NREL and Siemens Wind Power, CRADA no. CRD-08-303. The submitted paper has been offered by employees of the Alliance for Sustainable Energy, LLC (Alliance), a contractor of the U.S. Government under Contract No. DE-AC36-08GO28308. The U.S. Government retains a nonexclusive, paid-up, irrevocable, worldwide license to publish or reproduce the published form of this work, or allow others to do so, for U.S. Government purposes, and the publisher, by accepting the article for publication, acknowledges this.

Edited by: Gerard J. W. van Bussel

Reviewed by: two anonymous referees

\section{References}

Bayarri, M., Berger, J., Paulo, R., Sacks, J., cafeo, J., Cavendish, J., and Lin, C.-H.: A Framework for Validation of Computer Models, in: Technometrics, 49, 138-154, American Statistical Association and the American Society for Quality, https://doi.org/10.1198/004017007000000092, 2007.

Bergami, L. and Gaunaa, M.: Analysis of aeroelastic loads and their contributions to fatigue damage, Journal of Physics: Conference Series, 555, 012007, http://stacks.iop.org/1742-6596/555/i=1/a= 012007, 2014.

Blasques, J. P., Bitsche, R. D., Fedorov, V., and Lazarov, B. S.: Accuracy of an efficient framework for structural analysis of wind turbine blades, Wind Energy, 19, 1603-1621, https://doi.org/10.1002/we.1939, 2016.

Damiani, R., Haymany, G., Wangz, Q., Jonkman, J., and Gonzalez, A.: Development and Validation of a New Unsteady Airfoil Aerodynamics Model Within AeroDyn, in: 34th Wind Energy Symposium, American Institute of Aeronautics and Astronautics, San Diego, CA, 2016.

Guntur, S., Jonkman, J., Schreck, S., Jonkman, B., Wang, Q., Sprague, M., Hind, M., and Sievers, R.: FAST v8 Verification and Validation for a Megawatt-Scale Wind Turbine with Aeroelastically Tailored Blades, in: 34th Wind Energy Symposium, AIAA SciTech, San Diego, CA, USA, https://doi.org/10.2514/6.20161008, 2016.

Guzman, R. and Cheng, P.: Recommendations for load validation of an offshore wind turbine with the use of statistical data: ex- perience from alpha ventus, in: Journal of Physics: Conference Series, 749, The Science of Making Torque from Wind, 012017 , https://doi.org/10.1088/1742-6596/749/1/012017, 2016.

Hansen, M.: Aerodynamics of Wind Turbines, The Cromwell Press, UK, 1 edn., 2000.

IEC 61400-13: Wind turbine generator systems - Part 13: Measurement of mechanical loads, Tech. Rep. IEC 6140013, Geneva, Switzerland, http://infostore.saiglobal.com/store/ details.aspx?ProductID=658090, 2001.

Jonkman, J.: The New Modularization Framework for the FAST Wind Turbine CAE Tool, in: 51st AIAA Aerospace Sciences Meeting including the New Horizons Forum and Aerospace Exposition, American Institute of Aeronautics and Astronautics (AIAA), https://doi.org/10.2514/6.2013-202, 2013.

Kallesoe, B. and Kragh, K.: Field Validation of the Stability Limit of a Multi MW Turbine, Journal of Physics: Conference Series, 749, The Science of Making Torque from Wind, 042005, https://doi.org/10.1088/1742-6596/753/4/042005, 2016.

Medina, P., Singh, M., Johansen, J., Jove, A., Fingersh, L. J., and Schreck, S.: Inflow Characterization and Aerodynamic Measurements on a SWT-2.3-101 Wind turbine, in: 50th AIAA Aerospace Sciences Meeting including the New Horizons Forum and Aerospace Exposition, American Institute of Aeronautics and Astronautics (AIAA), https://doi.org/10.2514/6.2012230, 2012.

Ning, A., Hayman, G., Damiani, R., and Jonkman, J. M.: Development and Validation of a New Blade Element Momentum Skewed-Wake Model within AeroDyn, in: 33rd Wind Energy Symposium, American Institute of Aeronautics and Astronautics, https://doi.org/10.2514/6.2015-0215, 2015.

WTC Information Portal (FAST): https://nwtc.nrel.gov/FAST (last access: 25 August 2017), last modified: 19 March 2015.

Rasmussen, F., Hansen, M. H., Thomsen, K., Larsen, T. J., Bertagnolio, F., Johansen, J., Madsen, H. A., Bak, C., and Hansen, A. M.: Present Status of Aeroelasticity of Wind Turbines, Wind Energy, 6, 213-228, https://doi.org/10.1002/we.98, 2003.

Schepers, J., Boorsma, K., Cho, T., Gomez-Iradi, S., Schaffarczyk, P., Jeromin, A., Shen, W., Lutz, T., Meister, K., Stoevesandt, B., Schreck, S., Micallef, D., Pereira, R., Sant, T., Madsen, H., and Sorensen, N.: Final report of IEA Task 29, Mexnext (Phase 1): Analysis of Mexico wind tunnel measurements, Tech. Rep. ECNE-12-004, ECN, 2012.

Schepers, J., Boorsma, K., Gomez-Iradi, S., Schaffarczyk, P., Madsen, H., Soerensen, N., Shen, W., Lutz, T., Schulz, C., Herraez, I., and Schreck, S.: Final report of IEA Wind Task 29:Mexnext (Phase 2), Tech. Rep. ECN-E-14-060, ECN, 2014.

Sim, C., Basu, S., and Manuel, L.: On Space-Time Resolution of Inflow Representations for Wind Turbine Loads Analysis, Energies, 5, 2071, https://doi.org/10.3390/en5072071, 2012.

Simms, D., Schreck, S., Hand, M., and Fingersh, L.: NREL Unsteady Aerodynamics Experiment in the NASA-Ames Wind Tunnel: A Comparison of Predictions to Measurements, Tech. Rep. NREL/TP-500-29494, National renewable Energy Laboratory, 2001.

Skjoldan, P., Hansen, M., Rubak, R., and Thomsen, K.: Aeroelastic modal dynamics of wind turbines including anisotropic effects, Ph.D. thesis, 2011.

Snel, H., Houwink, R., Bosschers, J., Piers, W., van Bussel, G., and Bruining, A.: Sectional prediction of 3-D effects for stalled flow 
on rotating blades and comparison with measurements, Proc. of the European Community Wind Energy Conference, 395-399, 1993.

Sprague, M. A., Jonkman, J. M., and Jonkman, B.: FAST Modular Framework for Wind Turbine Simulation: New Algorithms and Numerical Examples, in: 33rd Wind Energy Symposium, American Institute of Aeronautics and Astronautics (AIAA), https://doi.org/10.2514/6.2015-1461, 2015.

Wang, Q. and Yu, W.: Variational-asymptotic modeling of the thermoelastic behavior of composite beams, Composite Structures, 93, 2330-2339, https://doi.org/10.1016/j.compstruct.2011.03.021, 2011.
Wang, Q., Johnson, N., Sprague, M. A., and Jonkman, J. M.: BeamDyn: A High-Fidelity Wind Turbine Blade Solver in the FAST Modular Framework, in: 33rd Wind Energy Symposium, American Institute of Aeronautics and Astronautics, https://doi.org/10.2514/6.2015-1465, 2015.

Wang, Q., Sprague, M., Jonkman, J., and Jonkman, B.: Partitioned Nonlinear Structural Analysis of Wind Turbines Using BeamDyn, in: 34th Wind Energy Symposium, American Institute of Aeronautics and Astronautics, San Diego, CA, 2016.

Zhang, P. and Huang, S.: Review of aeroelasticity for wind turbine: Current status, research focus and future perspectives, Frontiers in Energy, 5, 419-434, 2011. 